ACCEPTED MANUSCRIPT

\title{
Development of a coupled simulation toolkit for computational radiation biology based on Geant4 and CompuCell3D
}

To cite this article before publication: Ruirui Liu et al 2020 Phys. Med. Biol. in press https://doi.org/10.1088/1361-6560/abd4f9

\section{Manuscript version: Accepted Manuscript}

Accepted Manuscript is "the version of the article accepted for publication including all changes made as a result of the peer review process, and which may also include the addition to the article by IOP Publishing of a header, an article ID, a cover sheet and/or an 'Accepted

Manuscript' watermark, but excluding any other editing, typesetting or other changes made by IOP Publishing and/or its licensors"

This Accepted Manuscript is @ $\mathbf{2 0 2 0}$ Institute of Physics and Engineering in Medicine.

During the embargo period (the 12 month period from the publication of the Version of Record of this article), the Accepted Manuscript is fully protected by copyright and cannot be reused or reposted elsewhere.

As the Version of Record of this article is going to be / has been published on a subscription basis, this Accepted Manuscript is available for reuse under a CC BY-NC-ND 3.0 licence after the 12 month embargo period.

After the embargo period, everyone is permitted to use copy and redistribute this article for non-commercial purposes only, provided that they adhere to all the terms of the licence https://creativecommons.org/licences/by-nc-nd/3.0

Although reasonable endeavours have been taken to obtain all necessary permissions from third parties to include their copyrighted content within this article, their full citation and copyright line may not be present in this Accepted Manuscript version. Before using any content from this article, please refer to the Version of Record on IOPscience once published for full citation and copyright details, as permissions will likely be required. All third party content is fully copyright protected, unless specifically stated otherwise in the figure caption in the Version of Record.

View the article online for updates and enhancements. 


\title{
Development of a Coupled Simulation Toolkit for Computational Radiation Biology Based on Geant4 and CompuCell3D
}

\author{
Ruirui Liu ${ }^{1,5}$, Kathryn A. Higley ${ }^{1}$, Maciej H. Swat ${ }^{2}$, Mark A. J Chaplain ${ }^{3}$, Gibin G. Powathil ${ }^{4}$, James A. \\ Glazier $^{2}$ \\ ${ }^{1}$ School of Nuclear Science and Engineering, Oregon State \\ University, 100 Radiation Center, Corvallis, OR 97331, USA \\ ${ }^{2}$ Biocomplexity Institute, Indiana University, Bloomington, Indiana, USA
}

${ }^{3}$ School of Mathematics and Statistics, Mathematical Institute, University of St Andrews, St Andrews KY16 9SS, Fife, Scotland

${ }^{4}$ Department of Mathematics, College of Science, Swansea University, Swansea, SA2 8PP, United Kingdom ${ }^{5}$ Department of Radiation Oncology, Washington University School of Medicine, St. Louis, MO, 63110, USA

\section{Abstract}

Understanding and designing clinical radiation therapy is one of the most important areas of state-ofthe-art oncological treatment regimens. Decades of research have gone into developing sophisticated treatment devices and optimization protocols for schedules and dosages. In this paper, we presented a comprehensive computational platform that facilitates building of the sophisticated multi-cell-based model of how radiation affeets the biology of living tissue. We designed and implemented a coupled simulation method, including a radiation transport model, and a cell biology model, to simulate the tumor response after irradiation. The radiation transport simulation was implemented through Geant4 which is an open-source Monte Carlo simulation platform that provides many flexibilities for users, as well as low energy DNA damage simulation physics, Geant4-DNA. The cell biology simulation was implemented using CompuCell3D (CC3D) which is a cell biology simulation platform. In order to couple Geant4 solver with CC3D, we developed a "bridging" module, RADCELL, that extracts tumor cellular geometry of the CC3D simulation (including specification of the individual cells) and ported it to the Geant4 for radiation transport simulation. The cell dose and cell DNA damage 
distribution in multicellular system were obtained using Geant4. The tumor response was simulated using cell-based tissue models based on CC3D, and the cell dose and cell DNA damage information were fed back through RADCELL to CC3D for updating the cell properties. By merging two powerful and widely used modeling platforms, CC3D and Geant4, we delivered a novel tool that can give us the ability to simulate the dynamics of biological tissue in the presence of ionizing radiation, which provides a framework for quantifying the biological consequences of/radiation therapy. In this introductory methods paper, we described our modeling platform in detail and showed how it can be applied to study the application of radiotherapy to a vascularized tumor.

Keywords: Computational Radiation Biology, Geant4, CompuCell3D, RADCELL, Coupled Simulation, Microbeam Radiation Therapy, Vascular Tumor Simulation.

\section{Introduction}

Cancer is a significant health problem and is the leading cause of death associated with the aging of the population and lifestyle. Radiotherapy aims to sculpt the optimal dose distribution on the tumor volume while sparing healthy tissues. The benefits are threefold: patient cure or palliation, healthy tissue/organ preservation, and cost-efficiency [1]. However, there are some challenges that pose to radiation oncologists as they attempt to predict and interpret the biological consequences of radiotherapy treatment. The mathematical modeling, simulating mechanisms of radiation interaction with matter, serves a suitable approach to overcome these difficulties since modeling can help us predict biological effects of radiation. In terms of treatment planning in radiation therapy, modeling provides the means for optimizing radiation applications: the optimization methods implemented in treatment planning systems can be used to obtain the best achievable balance between the intended effects and inevitable side effects using quantitative mathematical models. Radiation-induced biological effects result from complex mechanisms that involve a multitude of processes running at very different spatial and temporal scales and need a multiscale modeling approach, such as modeling of radiation effects on subcellular scales, modeling of cell killing, and modeling biological effects in tissues or organs (reviewed by Friedland et al [2]).

However, this is a challenging task. Even using some rough mechanistic models for modeling radiation interactions with cells, such as the current linear-quadratic (LQ)-centered approaches, it remains quite difficult to extrapolate this at tissue level, considering the tumor heterogeneity and microenvironment of tumor. Alternatively, an integrated stochastic approach can be adopted to 
couple cell biology with radiation transport and has shown to quantify the biological consequences of radiation by taking into account the physical and biological processes of irradiated tumors at cellular level, which could be adopted to tackle the difficulties mentioned above.

Another aspect of modeling radiation-induced cellular effect needed to be mentioned is that modeling the proper spatiotemporal environment is crucial as a cell's behavior depends strongly on its neighboring cells. In other words, given the effects of radiation on a single cell, it is very hard to quantify the effects of these doses at the tissue scale. This is precisely a situation where multiscale quantitative tissue modeling can play an important role in designing better radiotherapy treatment protocols.

Recently, Powathil et al. developed a multiscale mathematical model of chemotherapy treatment, incorporating cell-cycle mediated intracellular heterogeneity and external oxygen heterogeneity to study the effects of cell-cycle, phase-specific chemotherapy, and its combination with radiation therapy [3]. In their model, the radiation dose was uniformly assigned to cells according to the prescription, rather than the radiation transport calculation to obtain cellular dose values. This is not an optimal approach as experimental results indicate that there is a stochastic distribution of cell dose after irradiation [4]. Although, some researches considered the stochastic characteristic of cell dose [5][6], the models for quantifying the radiation interactions in cell is simply relying on a Poisson distribution. It is not accurate enough to describe the radiation dose delivering mechanisms of different types of radiation as such models not only do not take into radiobiological parameters in cell kill mechanism, but also they do not distinguish between the radiation tracks by different types of radiation.

While there exist many models simulating the radiation induced cellular effects [7]-[12], very few offer the ability to study the impact of radiation on individual cell and yet, at the same time, give the ability to simulate entire tissue. CompuCell3D (CC3D) + RADCELL/Geant4 offers such capability. Our computational platform is based on two key software components: CC3D [13] and Geant4 [14]. Merging the two tools into one modeling platform gives us an ability to simulate dynamics of biological tissue is in the presence of ionizing radiation.

CC3D focuses on cell-based tissue models (where each biological cell is modeled as an individual entity). Simulations that focus on emulating cellular behavior can be divided into two broad categories: single-cell and multi-cell simulations. For single cell simulation, we implement detailed models of "intra-cellular dynamics" and typically this is accomplished by either using ordinary differential 
equations (reaction-kinetics models), reaction-diffusion equations (such as models implemented using Virtual Cell modeling platform http://www.vcell.org) or particle-based models (e.g., based on the MCell modeling platform http://mcell.org/) that are inspired by molecular dynamics models but are more coarse grained and operate at much larger time-scales.

While single cell-models are capable of explaining many phenomena observed at a single cell-level, the computational costs of running those models are very high, and consequently, building tissue models based on either of the approaches mentioned above are not practical. To deal with those computational limitations, multi-cell tissue models make a series of simplifying assumptions. The level of detail with which we represent a single cell in the multi-cell models usually determines the multi-cell model size (measured in terms of number of simulated cells). The simplest representation of single cell is a point in space (implemented in the Cellular Automata Model [15][16] or the Center Model [17]) allows representing relatively large number of cells in the simulations, while methods that aim to model cells as spatially extended domains capable of modeling cellular shapes and shape changes have to perform more computations. Yet, on modern computers, the more detailed multi-cell simulations such as those based on Cellular Potts Model (CPM) on which CC3D depends [18], or Subcellular Element Model [19] can model tissue fragments that are big enough for running fairly realistic virtual assays.

This is a methods paper where we present a novel computational framework that we have developed with a few example applications. It is worth noting that this paper is mainly focusing on its technical feasibility of implementing integrated simulation by combining RADCELL/Gean 4 and CC3D. The primary focus of this paper is on merging technique for coupling CC3D and Geant4, and the implementation of the developed platform for integrated modeling of temporal evolution of the cellular patterns after irradiation for achieving realistic, and predictive models of radiotherapy. The details of the modeling applications will be discussed in a subsequent paper.

In this paper, we firstly present how the simulation framework is developed. Particularly, the components of RADCELL, such as cell dose and DNA damage calculation, cell state transition model, coupling simulation technique between RADCELL/Geant4 and CC3D, etc., are introduced in methods and material section. Then we devote the last sections to present how the developed simulation framework is used to simulate the cell and tissue response after irradiation, and specifically, an example of vascular tumor irradiation using microbeam is studied. 


\section{Methods and Material}

The coupled simulation method includes radiation transport simulation, and cell biology simulation, to simulate the cell response after irradiation. The radiation transport simulation is implemented using RADCELL/Geant4, and cell biology simulation is implemented using CC3D. In the simulation, CC3D serves as a master control of the whole simulation process, and all the simulation process is controlled through the user interface of CC3D which supports Python scripting.

\subsection{CompuCell3D}

In our developed simulation platform, we use CC3D as our cell biology simulation platform, due to its easy implementation and powerful simulation capabilities. CC3D provides a user-friendly programming environment in order to develop simulation models. The fundamental structures and functions are developed using $\mathrm{C}++$ for speed, but in practice bulk of CC3D simulation specification takes place in Python which makes the daunting task of constructing the model much simpler. CC3D also provides a dedicated Model Editor (Twedit + ) that significantly reduces the burden of setting up the model by offering modelers with a set of code-assistants for the most common modeling tasks. In addition to simulating cellular mechanics, CC3D allows coupling of multiple modeling scales. It provides a set of PDE solvers that operate at tissue and organ scale while the reaction-kinetics solvers that CC3D provides (implemented using libroarrunner [20]) allow modeling of intracellularphenomena. By appropriate linking the modeling scales (from molecular to organ-level), we can build very sophisticated models of tissues and thus gain insight into how disease processes in tissues originate and progress [21]-[23].

CC3D implements CPM [18] also known as Glazier-Graner-Hogeweg Model (GGH) [24]-[27]. In CPM, we represent generalized cells as spatially-extended domains that reside on a lattice. Each generalized cell in CC3D is made up of lattice sites, which are referred to as pixels or voxels, and can represent biological cells, compartments of cells or other biological objects such as the extracellular matrix. Each lattice site is represented by a vector of integers $i$. When a pixel is part of a generalized cell, the cell index is referred as $\sigma(i)$, and the cell type is called $\tau(\sigma(i))$, shown as in Figure 1 . The description of interactions between generalized cells is implemented using the effective energy which determines many characteristics such as cell size, shape, motility, adhesion strength, and the reaction to gradients of chemotactic fields. 
(a)

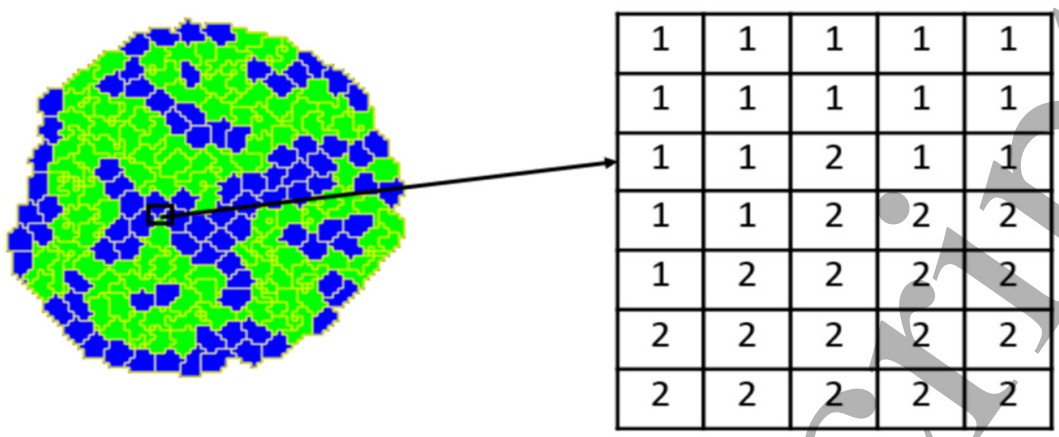

(b)

\begin{tabular}{|c|c|c|c|c|c|c|c|c|c|c|}
\hline \multirow{4}{*}{\multicolumn{2}{|c|}{ Changed pixe }} & & & & & 1 & 1 & 1 & 1 & 1 \\
\hline & & & & & & 1 & 1 & 1 & 1 & 1 \\
\hline & & & & & Index-copy succeeds & 1 & 1 & 1 & 1 & 1 \\
\hline & & & & & or $P=e^{-\Delta \mathrm{H} / T_{m}}: \Delta \mathrm{H}$ & 1 & 1 & 2 & 1 & 1 \\
\hline & & & & & & 1 & 1 & 2 & 2 & 2 \\
\hline 1 & 1 & 1 & 1 & 1 & & 1 & 2 & 2 & 2 & 2 \\
\hline 1 & 1 & 1 & 1 & 1 & & 2 & 2 & 2 & 2 & 2 \\
\hline 1 & 1 & 1 & 1 & 1 & & & & & & \\
\hline 1 & 1 & 1 & 1 & 1 & & 1 & 1 & 1 & 1 & 1 \\
\hline 1 & 1 & 2 & 2 & 2 & & 1 & 1 & 1 & 1 & 1 \\
\hline 1 & 2 & 2 & 2 & 2 & & 1 & 1 & 1 & 1 & 1 \\
\hline 2 & 2 & 2 & 2 & 2 & & 1 & 1 & 1 & 1 & 1 \\
\hline & & & & & & 1 & 1 & 2 & 2 & 2 \\
\hline & & & & & & 1 & 2 & 2 & 2 & 2 \\
\hline & & & & & & 2 & 2 & 2 & 2 & 2 \\
\hline
\end{tabular}

Figure 1: Detail of a typical two-dimensional CPM cell-lattice configuration. Each colored domain represents a single spatially-extended cell. The detail shows that each generalized cell is a set of cell-lattice sites, $i$ with a unique index, $\sigma(i)$ here 1 or 2 . The color denotes the cell type, $\tau(\sigma(i))$. CPM representation of an indexcopy attempt for two cells on a 2D square lattice- The "grey" pixel (source) attempts to replace the "white" pixel (target). A pixel, chosen at random, of type 2 cell makes a pixel-copy attempt to a neighboring pixel of a type 1 cell. The pixel-copy attempt is accepted and the neighboring pixel transitions from type 1 to type 2 . If the attempt is not accepted, then there will be no pixel transitions.

A simulation progresses by attempts of generalized cells to extend their boundaries in an effort to minimize the effective energy. These are called index-copy attempts because they try to change the cell index of a neighboring pixel to that of its cell type. The success of the index copy attempt is dependent upon a Boltzmann acceptance function which takes into account the change in energy, which is illustrated in Figure 1. The detailed description of the effective energy calculation and the 
simulation dynamics is referred to section 1 of supporting document: CompuCell3D general introduction.

\subsection{Geant4 in General}

Geant4 is a freely available software for performing Monte Carlo simulations of the interactions of energetic particles in matter [14]. In the developed simulation platform, Geant4 serves as the radiation transport solver for our specific simulations, such as calculating the energy deposition points inside cells. The main reason for choosing Geant4 is that it is an open-source Monte Carlo simulation platform, and it provides many flexibilities for users, and also the low energy DNA damage simulation physics process, Geant4-DNA.

\subsection{RADCELL}

In order to couple Geant4 solvers with $\mathrm{CC} 3 \mathrm{D}$, we need a "bridging" code that extracts current geometry of the CC3D simulation (including specification of the individual cells) and ports it to a Geant4 solver. In other words, we need to "translate" representation of biological cell as implemented in CC3D into a computational representation of the cell that Geant 4 can work with. In the meantime, the simulated cell dose and DNA damage information needed to be fed back to CC3D to update the cell properties. This task is accomplished using our newly developed module, RADCELL. RADCELL is a radiation transport simulation module developed for conducting radiation transport simulation in cells. It can simulate the cell dose and cell DNA damages, such as single-strand breaks (SSBs) and double-strand breaks (DSBs). The RADCELL is developed based on the microdosimetry example of Geant4. The functionalities of RADCELL are referred to section 2 of supporting document: RADCELL general introduction.

The primary function of RADCELL is to calculate the radiation dose to cell organelles, and DNA damages to cells. In this work, we propose a three-dimensional (3D) cellular compartment model, which incorporates two cellular compartments including nucleus and cytoplasm. We use the sphere to approximate the cell shape which substantially simplifies the complexity of cell geometry but there is no significant big accuracy penalty [28]. The nucleus is modeled as a sphere which is located at the center of the cell. The size of the cell and the nucleus can be customized according to the biological cell which will be studied during the simulation.

During the radiation transport simulation, the energy deposition information in each cell will be collected, and the information, such as the index for indicating the affected cells, cellular dose, and affected cell organelles, etc., could be used to quantify the cell dose and DNA damages. 


\subsubsection{Cell Dose Tally and DNA Damage Tally}

After we obtain the energy deposition information in each event, then we process these data to obtain the cell dose tally and cell DNA damage tally. In RADCELL, cell dose tally is quantified by summing the total energy deposited in cell and dividing the energy deposited by cell mass. For cell DNA damage tally, the SSB and DSB yields are quantified using a clustering algorithm, i.e., DBSCAN (Densitybased spatial clustering of applications with noise) [29] for processing the energy deposition information inside nucleus. The clustering algorithm is a popular method for quantifying DNA damages yield, which is discussed in [30]-[32]. A detailed description of the development of the algorithm for cell dose tally and DNA damage tally based on Geant 4 simulation is discussed in our previous work [33][34]. In the current functionality for modeling radiation-induced DNA damage in RADCELL, there are a few limitations needed to be mentioned. For the DNA damage repair of cell, we don't simulate the DNA damage repair and aberrant repair, etc. Modeling approaches based on biochemical kinetic equations have been proposed for representing the course of the base and nucleotide excision repair systems, which is used by cells to remove base damages and strand breaks and more bulky lesions, respectively [2]. The DSB damage repair mechanism, such as nonhomologous end joining (NHEJ) could be modeled to simulate the DSB repair in cell [35]. We don't simulate the radiation-induced DNA damages of mitochondria, and this may underestimate the total DNA damages of radiation to some cell lines [36][37]. Due to the easy extensibility of RADCELL/Geant4, extra models can be added to address these issues.

\subsubsection{Cell State Transition Model}

In this work, we use a cell state model to quantify the temporal transition of the possible cell phenotypes after irradiation [38]-[40]. We defined three major cell states: Healthy, Arrested, and Dead [34]. A Healthy cell maintains its basic functionality or keeps a proliferative state with no or very light damage. An Arrested cell has its cycle halted in a specific cell-cycle phase. A Dead cell has suffered irreparable damage and suspends material exchange with extracellular matrix (ECM). Each of these cell states differs depending on the cell's phase in the cell cycle: $\mathrm{G}_{1}, \mathrm{~S}, \mathrm{G}_{2}$, or M. The allowed state transitions are:

- From Healthy to Arrested or Dead.

From Arrested to Dead or Healthy.

Dead cells stay Dead. Transitions depend only on a cell's current state. 
These state transition rules are based on radiation biology experiments. For instance, 1) A high dose causing direct cell death corresponds to the state transition from Healthy to Dead; 2) A moderate dose causing cell-cycle arrest corresponds to the state transition from Healthy to Arrested; 3) Cell apoptosis after failed cell damage repair during cell-cycle arrest, corresponds to the state transition from Arrested to Dead; 4) A dead cell does not have capacity to repair damage, so the,Dead state is persistent. The mathematical description of the cell state transition model is referred to section 3 of supporting document: Cell State Transition Model.

During simulation, the cell state transition is taken as a continuous stochastic process evolving with time after cell irradiation. Firstly, the external perturbation energy $\Delta E$ for each cell is updated, then the probability of corresponding cell state transition is calculated based on $\Delta E$ in the time step. Secondly, the cell state transition decision is made based on the calculated transition probability according to rejection sampling rule [41]. An example of updating the cell state in one time step is shown in Figure 2. Basically, the cell phase transition and cell state transition of cell after irradiation are followed and updated using a cellular automaton method.

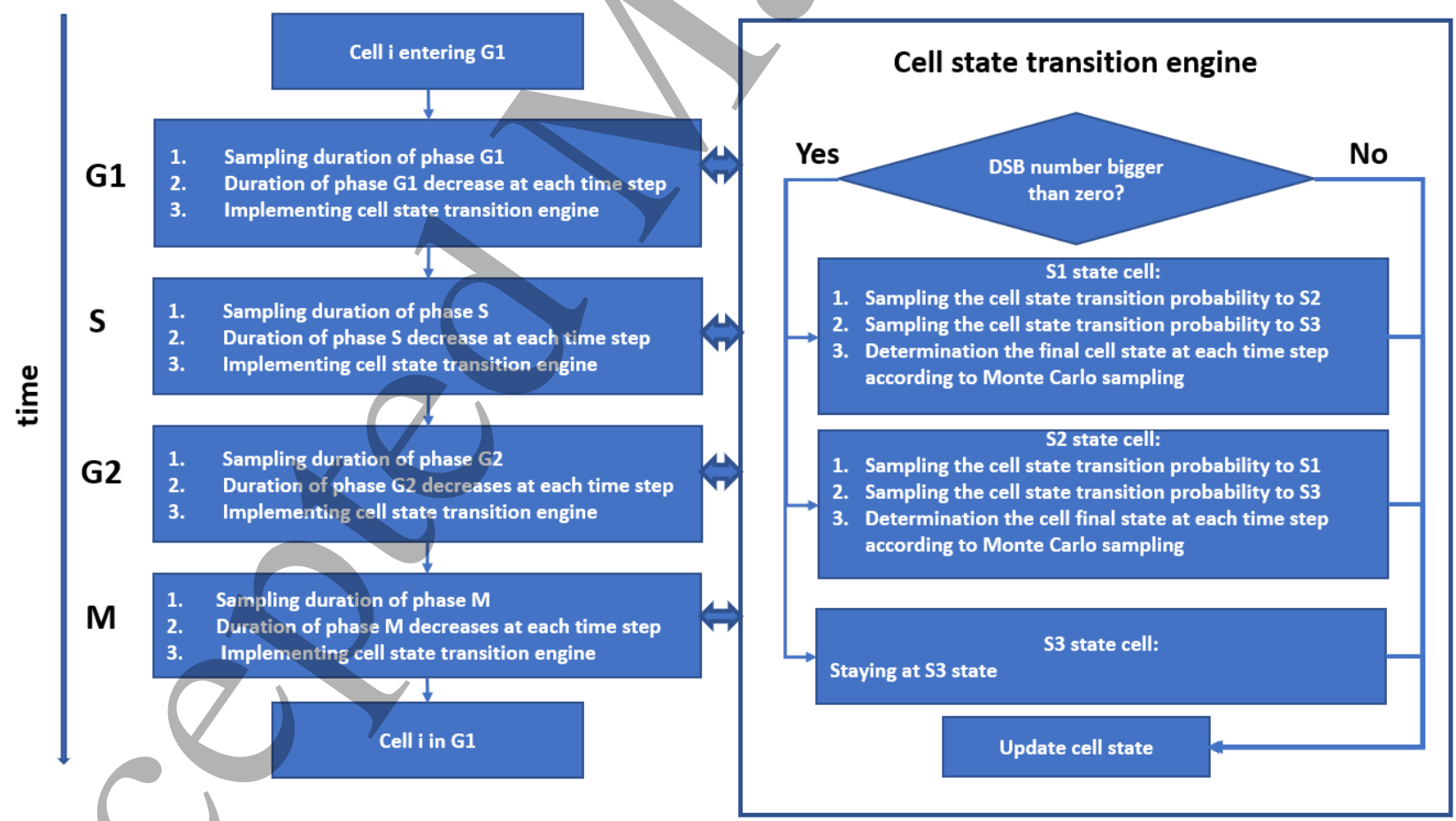

Figure 2: Process of updating cell state in one time-step. During simulation, the state of all the cells will be updated in each time step which could be defined in the simulation. Cell phase transition is modeled by comparing the sampled cell phase duration and time of cell staying in the cell phase. If the time of cell staying is larger than the sampled cell phase duration, then cell phase transition will occur. If mitosis occurs, 
then two new cells will be added to the cell system. Cell state transition in each cell phase are also modeled through the defined cell state transition rules. In each time step, the cell phase and cell state transition are updated.

\subsection{Coupled Simulation Using RADCELL and CompuCell3D}

To enable seamless integration of RADCELL, Geant4 and CC3D, we have developed Python modules that allow using of all RADCELL functionalities from the Python level, thus making it very easy to integrate with CC3D. Geant 4 is a pure $\mathrm{C}++$ platform, and there is very little python development, Geant4Py, which is based on Boost-C++ external library [42]. However, this has not been maintained and developed for a long time and it only has very limited functionality for the users. So, in order to use Geant4 in CompuCell3D which is a pure python-based platform for regular users, we have to develop a new and efficient bridge software for using Geant4 in python. In our study, we developed a bridging module based on SIWG [43], and this enable us to use all the functionality of Geant4 in a python environment. Therefore, we can quantify cell dose and cell DNA damages and use those quantities to determine the cell state transitions for CC3D cells. This is significant, because using our approach, we can have the most up-to-date information about cell irradiation and thus can simulate with great level of detail how radiation impacts cell cycle or any other cell properties that can potentially be affected by increased levels of radiation.

\subsubsection{Importing Cells into Geant4 from CC3D}

Any attempt to couple separate simulators (in this case, Geant4 and CC3D) requires development of systematic methods that allow transferring information about simulated objects between the separate simulators. In our case, the challenge is how to represent CC3D cells in Geant4. To do that, we first extract CC3D cell position information and pass this information to Geant4. We then create Geant4 equivalents of CC3D cells in such a way that relative cell distances are preserved in Geant4. Getting correct positions of cells is crucial if we want to obtain the accurate radiation dose distribution for all the cells.

In Geant4, the cell has the same size as the biological cell for modeling the physical interactions accurately. The cells in Geant4 are seeded according to cells' physical sizes. In this iteration of our software, we make a simplifying assumption about Geant 4 cell shape and treat all the cells as spheres. After obtaining the cell position and size information, the cells are seeded into Geant 4 for radiation transport simulation. 
One of the potential problems is that the cells in Geant4 may overlap with each other if we simply seed the cells according to the center of mass (COM) of cells in CC3D. To overcome this problem, we keep the size of Geant 4 cell constant and rescale the whole geometry to the point where all Geant4 spheres representing CC3D cells are non-overlapping. This rescaling has negligible effect of assessment of radiation damage. One way to think about it is to imagine that after this rescaling, the Geant4 spheres represent the entire CC3D cell but with a somewhat smaller CC3D cell volume (one that includes cell's nucleus) that is directly susceptible to radiation effects.

Another thing to consider is the cell motion. Since CC3D is a Monte Carlo simulation technique, in every Monte Carlo Step (MCS) a given cell will appear in slightly different position. However, physically observed cell displacement are those that are observed every several MCS (the exact number of MCS interval depends on the simulation parameters [13]). We thus set a predefined MCS interval and use it to synchronize CC3D and Geant4 tissue layouts.

\subsubsection{RADCELL, CC3D, and Geant4 interoperability}

After the radiation transport simulation (using Geant4), the cell dose information and cell DNA damage information are written to CSV files respectively. Then CC3D will read the dose and DNA damage information when they are needed for determining the cell state transition after irradiation. In the next releases of our software, we will eliminate the need to carry those CSV files and exchange information between Geant4 and CC3D using a more elegant solution.

When we run the simulation, CC3D is the master module that controls the execution of RADCELL/Geant4, as shown in Figure 3. The CC3D simulation (all internal modules of CC3D) is implemented in one operating system process, while the radiation transport simulation (using Geant4) uses another process which is different from the CC3D's process.

As shown in Figure 3, at $\mathrm{n}^{\text {th }}$ MCS, in CC3D, RADCELL is launched to run the radiation transport simulation, so the radiation transport simulation results are just for the cells at $\mathrm{n}^{\text {th }}$ MCS. It is essential to ensure that while RADCELL (Geant4) executes radiation transport calculations, CC3D waits for the radiation transport results before proceeding further. Once $\mathrm{CC} 3 \mathrm{D}$ gets radiation information, it will modify cell properties accordingly and proceed with the next $n+1 \mathrm{MCS}$, and the whole process of interleaved CC3D+ RADCELL execution will continue to repeat until CC3D simulation is over. 


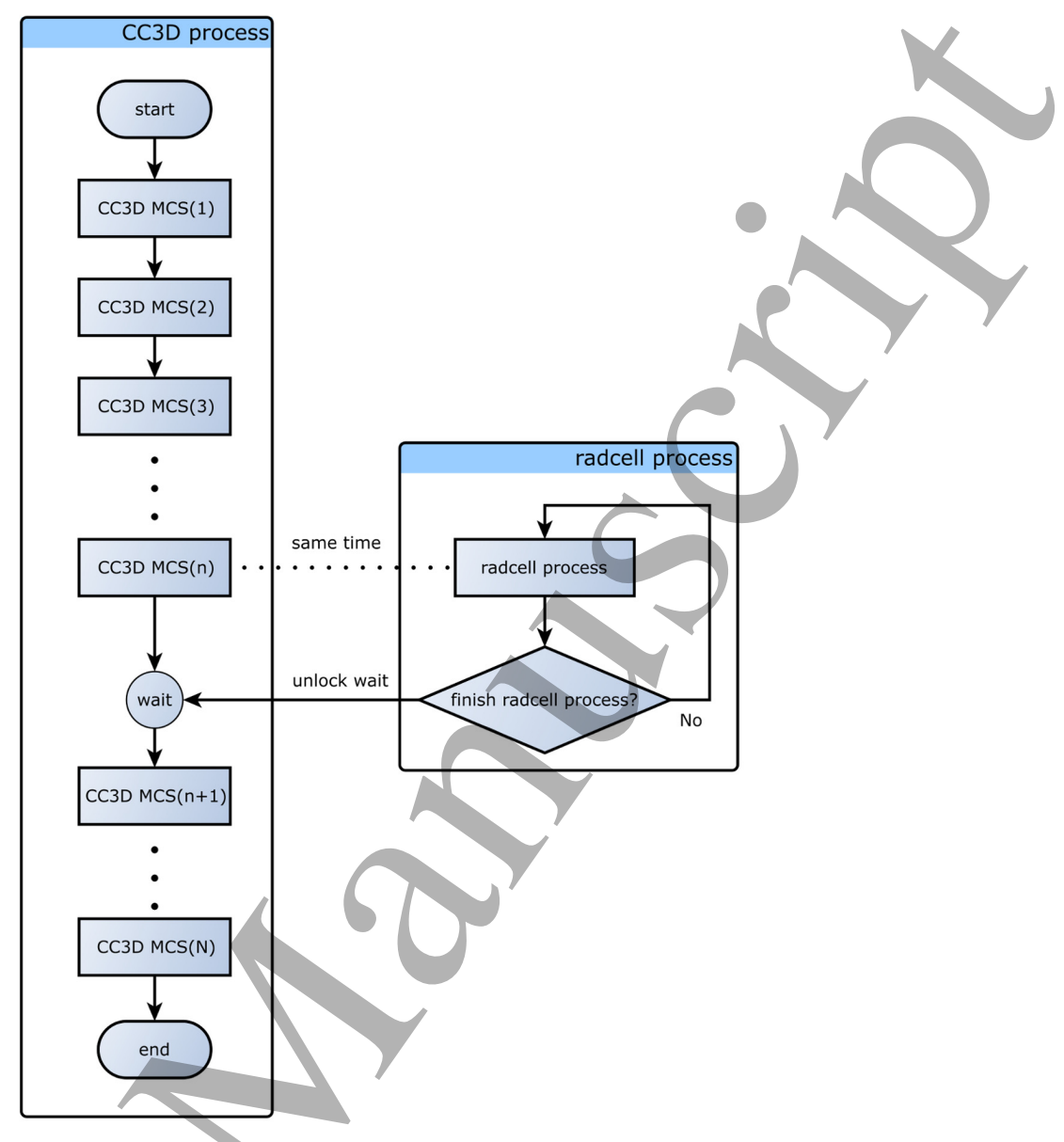

Figure 3: The coupled simulation process between RADCELL and CC3D.

\subsection{Code repository}

In order to achieve code reuse, we share the code including the parameter and document in an opensource software repository (https://github.com/forgetsummer/RADCELL). CompuCell3D and Geant 4 are required to run the simulation and are available for download.

\section{Example Model}

In this section, we talk about how to use the developed simulation platform to simulate the cell and tissue response after irradiation. We developed a simplified model of microbeam irradiation of a vascularized tumor. Our developed simulation platform is a suitable for modeling the vascular tumor response from microbeam irradiation. CompuCell3D is one the state-of-the-art techniques for modeling the tumor characteristics, and it has been widely used in simulating the tumor characteristics, such as the modeling the tumor growth and angiogenesis [44], cancer metastasis [45], stratification in solid tumors [22], and vascular tumor angiogenesis [27]. It is computationally 
restricted to model a fully grown tumor which has gigantic number of cells from multicellular perspective. One common strategy for modeling radiation therapy is modeling the radiation response for a small tumor spheroid which is relatively easy to handle through multicellular modeling [46][48]. In the microbeam therapy, the spatial scale of radiation beam of the microbeam matches with the spatial size of a spheroid model of vascular tumor. Thus, RADCELL/Geant4 is suitable platform for simulate the radiation transport of the vascular tumor spheroid through multicellular simulation so as to get the explicit cellular dose distribution and cellular DNA damage distribution.

This model is provided as a demonstration of the capabilities of CC3D-RADCELL/Geant4 modeling platform. The typical Microbeam Radiation Therapy (MRT) treatments deliver microscopically discrete spatial dose distributions: 10-100 microns wide parallel beams with a separation of several hundred microns between adjacent beams. Because high-dose, high-precision MRT's have potential to significantly reduce probabilities of healthy tissue complications [49][51], they are considered as a promising treatment concept. However, one of the significant challenges is the lack of comprehensive understanding of the underlying radiobiological mechanism. It is commonly acknowledged that MRT is based on the principle that healthy tissues can tolerate high doses of radiation in small volumes and that MRT can damage the blood vessels, cut off the tumor nutrient supply, and as a result, cause the tumor to die [52]-[54].

To better understand the radiological mechanism of MRT, we focus on two core areas: 1) understanding how radiation transport process of microbeam radiation affects tumor and healthy tissues, 2) understanding the tumor response after microbeam irradiation.

While microbeam radiation transport has been already addressed in several publications, e.g., [55][56], there has been less emphasis on how tumor behaves following MRT. Because the RADCELL-CC3D platform facilitates coupled tissue-radiation simulations, it is an ideal tool to conduct such studies.

In the remainder of this section, we present a detailed guide demonstrating all steps needed to build a model of radiation treatment of vascularized tumor. Our model simulates essential cell behaviors, microenvironmental components, and their interactions as well as radiation response after irradiation. The basic modeling methods and procedures are discussed as follows. The work is based on a simulation work by Swat et al. [27]. The sub-models simulated in the example simulation are displayed in Table 1. It is worth noting that these models are just selected for demonstrating the capability of coupled simulation of our developed simulation framework. Due to the easy extensibility, other models could be easily added for achieving more functionalities. 
Table 1: Models simulated in simulating the MRT treatment of vascular tumor

\begin{tabular}{|c|c|c|c|}
\hline \multicolumn{2}{|c|}{ Sub-models } & Functionality & Platform used \\
\hline \multirow{4}{*}{$\begin{array}{l}\text { Cell } \\
\text { biology } \\
\text { modeling }\end{array}$} & Multi-cellular model & Building tumor cells & CC3D \\
\hline & Reaction-diffusion model & $\begin{array}{l}\text { VEGF signaling process; glucose } \\
\text { reaction-diffusion process }\end{array}$ & \\
\hline & Cellular Potts Model & $\begin{array}{l}\text { Cell-cell adhesion; cell volume } \\
\text { constraint; cell surface constraints }\end{array}$ & CC3D \\
\hline & $\begin{array}{l}\text { Michaelis-Menten expression } \\
\text { from enzyme kinetic model }\end{array}$ & Cell growth and mitosis & CC3D \\
\hline $\begin{array}{l}\text { Radiation } \\
\text { transport } \\
\text { modeling }\end{array}$ & Radiation transport model & $\begin{array}{l}\text { Parallel electron microbeam } \\
\text { transport in tissue }\end{array}$ & RADCELL/Geant4 \\
\hline
\end{tabular}

\subsection{Vascular Tumor Model in CC3D}

Firstly, we use CC3D to build the vascular tumor model. An initial condition for our vascularized tumor model is a cluster of proliferating tumor cells and a simple network of pre-existing normal vasculature. Initially, tumor cells proliferate as they take up diffusing glucose which the pre-existing vasculature supplies. In this work, we expect that the tumor cells (both in the initial cluster and later) are usually hypoxic and they can secrete a long-diffusion isoform of VEGF-A which is denoted as LVEGF here. When the concentration of glucose drops below a threshold, tumor cells end up necrotic, step by step shrink, and ultimately disappear. A few preselected neovascular endothelial cells in the pre-existing vasculature respond both via chemotaxing toward greater concentration of proangiogenic factors and form new blood vessels by means of neoangiogenesis. The preliminary tumor cluster grows and reaches a maximum diameter characteristic of an avascular tumor spheroid. When the tumor grows to a certain size, we simulate the application of microbeam irradiations that kills certain fraction of tumor and vascular cells. We simulate deliveries of different irradiation schemes and implement basic mechanisms that simulate tumor response and evolution after irradiation.

\subsubsection{Cell and ECM Types}

Our model of solid tumor includes two main classes of generalized cells: tumor cells and stromal tissue. In this work, we stipulate four cell types: P: proliferating tumor cells; N: necrotic cells; EC: endothelial cells; NV: neovascular endothelial cells and ECM: an aggregate of stromal cells and ECM. This model focuses on small, early-stage vascular tumors rather on developed primary tumors, to identify the pattern of cell-behavior selection. While the average number of cells is far less than a real vascular tumor at any time, we can map these simplified model tumors onto real tumors either by 
considering each model cell to represent an ensemble of hundreds or thousands of real cells or by considering each model tumor to represent a peripheral microportion of a much larger tumor mass. In CC3D, we set the cell and field lattice dimensions to $50 \times 50 \times 80$, the membrane fluctuation amplitude to 20 , the pixel-copy range to 3 , the number of MCS to 17,000 , and choose UniformInitializer to produce the initial tumor and vascular cells, since it automatically creates a mixture of cell types.

\subsubsection{Chemical Fields}

In this study, we consider two types of chemical fields, i.e., glucose, and VEGF-mediated signaling factors. The cluster of tumor cells form a spheroid, the nutrient and waste diffusion will limit the diameter of such avascular tumor spheroids to about $1 \mathrm{~mm}$. The eentral region of the developing spheroid will become necrotic, with a surrounding layer to cells whose hypoxia triggers VEGFmediated signaling events that initiate tumor neovascularization by way of promoting growth extension or nearby blood vessels [27]. We add a set of finite-element links between the EC cells to model the strong junctions that form between EC cells and NV cells chemotax up gradients of two diffusing isoforms of VEGF-A, i.e., S-VEGF and L-VEGF. Both EC cells and NV cells chemotax up S-VEGF, but only NV cells chemotax up gradients of L-VEGF. We assume that glucose includes a diffusing field representing glucose. The L-VEGF and S-VEGF include diffusion fields representing L-VEGF and S-VEGF respectively.

\subsubsection{Cell Interactions}

Following basic assumptions of the CPM model [18], we simulate cell interactions by specifying effective energies associated with particular cell behaviors. The detailed description of cell interactions is referred to section 4 of supporting document: Cell Models Used in CC3D Modeling.

\subsubsection{Cell Growth and Mitosis}

In this study, we assume that glucose is the main growth-limiting substance for tumor cell. The LVEGF is the main growth-limiting substance for neovascular cell. The concentration rate of glucose could be described by the reaction-diffusion equation. Cell growth and contact-inhibition growth of NV cells are modeled in this work. The detailed description of the models could be referred in section 4 of supporting document: Cell Models Used in CC3D Modeling.

\subsection{Radiation Transport Using RADCELL}

In our coupled simulation, we import RADCELL directly into CC3D as a Python module. The radiation simulation is interleaved with the simulation of cellular pattern evolution. After each 
application of radiation in our model, we query the RADCELL module to provide information about cell dose and DNA damages for every biological cell present in our models.

We use cell dose and cell DNA damage information to determine the cell state transition in CC3D which typically amounts to altering cell parameters such as cell type, cell target volume, and cell target surface. In general, every parameter that describes cellular behavior can be altered, and it is up to a modeler to come up with a reasonable way of doing it.

The value of combining radiation transport simulation within $\mathrm{CC} 3 \mathrm{D}$ is that we can obtain the most up-to-date information about radiation damage and consequently build a more realistic radiation treatment model.

\subsubsection{Radiation Source}

To model the MRT irradiation, we chose a square plane with $50 \mu \mathrm{m}$ width as a radiation source. This dimension is in line with the width of the parallel microbeam used in MRT. Typical energies of X-ray radiation used in MRT range from $50 \mathrm{keV}$ to $600 \mathrm{keV}$. Considering that the dose of X-ray is induced by the secondary electrons, we make a simplifying assumption and use $600 \mathrm{keV}$ electron to model the $600 \mathrm{keV} \mathrm{X}$-ray for the sake of computational speed and efficiency. It is worth noting that this is a simplification, but it should give us a good "first-order approximation" of the real phenomena.

\subsubsection{Importing Cells into Geant4 from CC3D}

During the simulation of radiation transport, we extract the cell position in CC3D and pass this information to Geant4 to create proxies of CC3D cells in Geant4 simulator. Since we preserve the geometry of the CC3D tissue, the information about radiation damage we get from RADCELL/Geant4 should be accurate enough that we can assess radiation effects on each cell.

\subsection{Cell State Transition}

In our simplified model, we consider two cell states, i.e., healthy state $\left(S_{1}\right)$ and dead state $\left(S_{3}\right)$. We define the cell state transition rule according to two types of conditions of cell, i.e., microenvironmental factors and irradiation. Experimentally, microenvironmental factors, including mechanical stress, hydrostatic pressure, low $\mathrm{pH}$, and starvation, can cause temporary or permanent changes in tumor cells. In this model, however, we only include cell state transitions due to nutrient availability and radiation dose. During a period of starvation (in a low-nutrient regime), if the glucose concentration is lower than the threshold, then tumor cell dies. For nutrient availability condition, we simply calculate the glucose concentration for tumor cells at each MCS and compare 
the concentration with the threshold concentration. For quantifying the cell state transition after irradiation, we only consider the direct effect of irradiation. The cell state transition rule for glucose concentration and irradiation is referred to section 5 of supporting document: Cell State Transition.

\subsection{Simulation Parameter}

CPM simulations measure simulation time in terms of MCS, and the conversion between MCS and experimental time depends on the average cell motility. Biologically, MCSs are proportional to the experimental time [22]. We can relate the simulation's MCS time-scale to minutes by comparing cellmigration speeds in simulation to typical cell-migration speeds in experiments. In this study, we use a 3D voxel with a side of $4 \mu \mathrm{m}$, so the tumor cell volume is $64 \mu \mathrm{m}^{3}$. Since the experimental human umbilical vein endothelial cells (HUVEC) speed is about $0.4 \mu \mathrm{m} / \mathrm{min}$ [27], and the cells in this simulation move at an average speed of $0.1 \mathrm{pixel} / \mathrm{MCS}$, so one MCS represents 1 minute. Here, by knowing the time scale conversion between the CC3D time scale and the experimental time scale, we use MCS as the time unit for all the parameters related to time hereinafter in this work. The simulation parameters are referred to section 6 of supporting document: Simulation Parameters.

\subsection{Simulation Results}

\subsubsection{Tumor growth Without Irradiation}

We run otherwise identical simulations with and without MRT irradiation to study how MRT irradiation affects tumor growth and morphology. To begin with, we run the simulation to see how the tumor system will evolve without irradiation. The simulation results serve as a control for evaluating the irradiation effectiveness of MRT.

As shown in Figure 4b, we initialize the tumor cell cluster and two crossing vascular cords. We also add two NV cells to each vascular cord, 25 pixels apart. Without irradiation, the tumor system follows its typical biological evolution path, and we can see that the tumor grows bigger and bigger with the glucose update. We evaluate tumor growth by analyzing the number of proliferating tumor cells with respect to time. The simulation of tumor growth without irradiation showed an apparent increase of proliferating tumor cells with time, as shown in Figure 4a. The period dips reflect the cell loss due to the necrotic cell death because of the nutrient deficiency. This is in line with the biological observations in experiments, that the nutrient diffusion limits the diameter of the cluster of tumor cells, and the central region of the growing tumor becomes necrotic [27]. Though initially we only seed two NV cells, we can observe that the neovascular cells are undergoing proliferation with the simulation time going on, and this is triggered by VEGF-mediated signaling events that 
initiate tumor neovascularization by promoting growth and extension (neoangiogenesis) of nearby blood vessels.

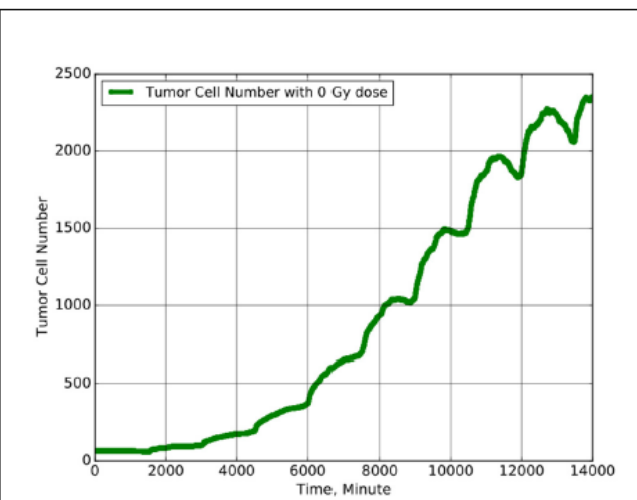

(a) Number of proliferating tumor cell with respect to time.

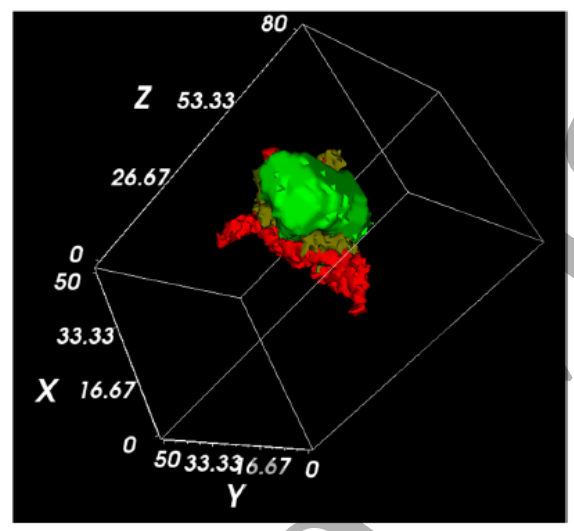

(c) Tumor system at $4000 \mathrm{MCS}$

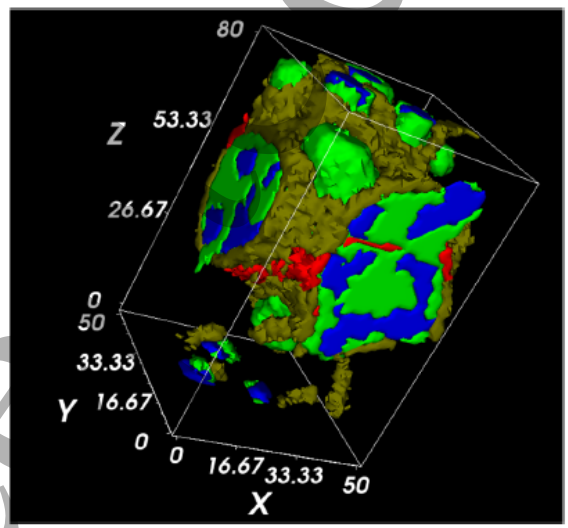

(e) Tumor system at $11000 \mathrm{MCS}$

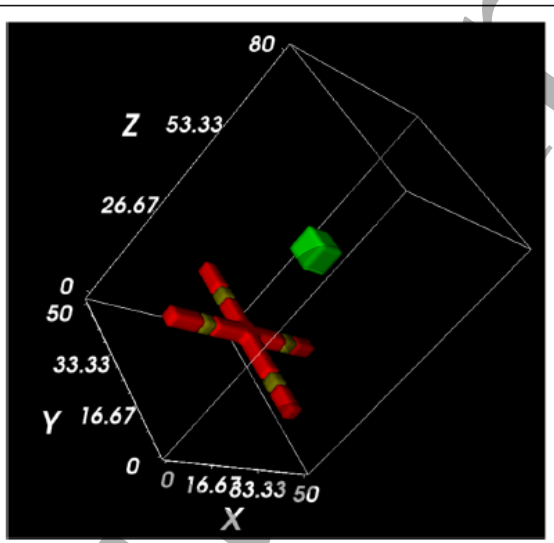

(b) Tumor system at $0 \mathrm{MCS}$

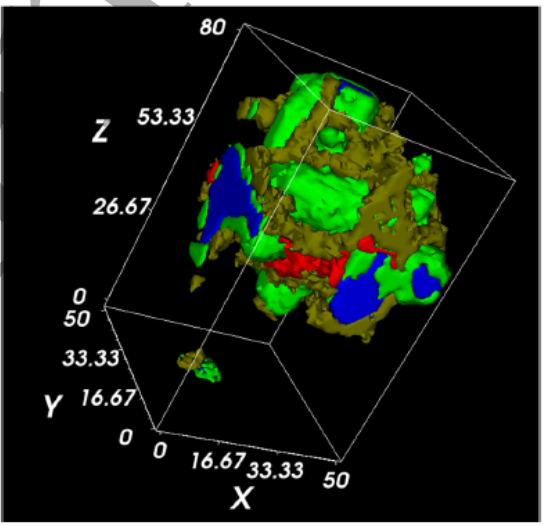

(d) Tumor system at $9000 \mathrm{MCS}$

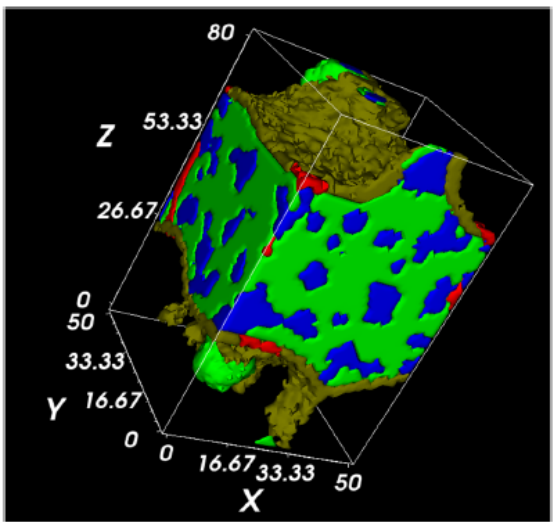

(f) Tumor system at $14000 \mathrm{MCS}$

Figure 4: Two-dimensional snapshots of the vascular tumor simulation. Red: Vascular cells, grey:

neovascular cells, blue: necrotic cells. The spatial unit used in CC3D is pixel, and one pixel equals $4 \mu \mathrm{m}$ in this work. Fig. (a) shows the tumor growth curve with respect to tumor evolution time which has MCS as its 
unit. Fig. (b) shows tumor system at 0 MCS (starting time) rendering in CC3D visualization system. Figs. (c), (d), (e), and (f) show the tumor system at 4000, 9000, 11000, and 14000 MCS, respectively.

\subsubsection{Tumor Growth with Irradiation}

For radiation therapy, radiation could be considered as an external perturbation agent to the tumor system. As we just simulate, without irradiation, the tumor system follows an unperturbed biological growing path, which indicates that the CC3D model can capture the basic biological characteristics of vascular tumor growth. Knowing this, we are more interested in knowing how the vascular tumor respond to the irradiation. Here, we conduct a simulation for simulating the tumor growth under different irradiation schemes.

In this simulation, we use a single planar microbeam source to irradiate the vascular tumor from an early time (starting from $1000 \mathrm{MCS}$ ), and the dose is delivered in five fractions equally. The total dose is from 5 Gy to $30 \mathrm{~Gy}$. The dose delivering time is at: $1000 \mathrm{MCS}, 5000 \mathrm{MCS}, 7000 \mathrm{MCS}$, $9000 \mathrm{MCS}$, and $11000 \mathrm{MCS}$.

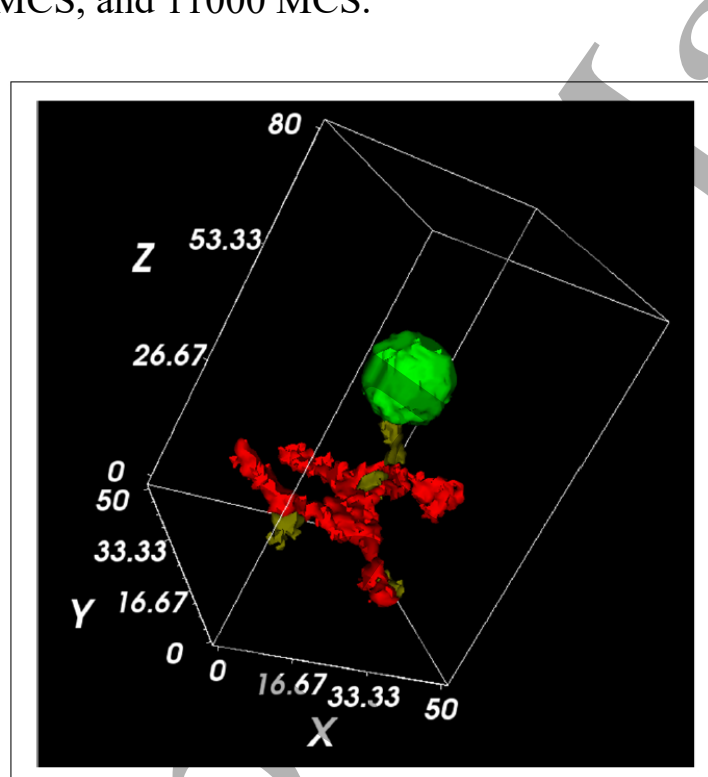

(a) At 1000 MCS in CCD visualization

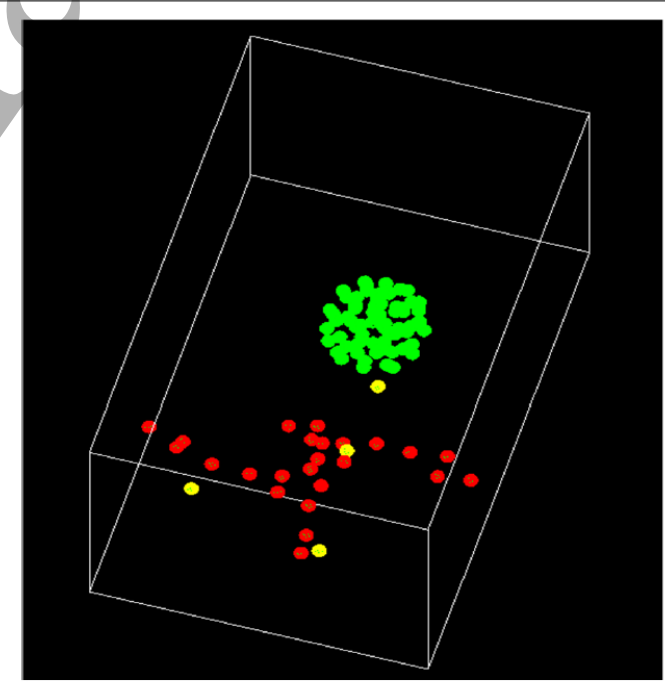

(b) At 1000 MCS in Geant4 visualization

Figure 5: Two-dimensional snapshots of the vascular tumor simulation. The spatial unit used in CC3D is pixel, and one pixel equals $4 \mu \mathrm{m}$ in this work. Fig. (a) is the tumor visualized in CC3D, and Fig. (b) is the tumor visualized in Geant4. Red: vascular cells, grey: neovascular cells, blue: necrotic cells.

The vascular tumor at 1000 MCS is a relatively small cluster, as shown in Figure 5a. During simulation, when a vascular tumor evolves to this time, CC3D automatically launches the radiation transport simulation by calling the functions in the RADCELL module. The tumor geometry is 
extracted from CC3D and imported to Geant4 for radiation transport simulation, as shown in Figure $5 b$.

It is worth noting that the materials of all the cells and the materials between cells are modeled as water in RADCELL. Each time after radiation transport simulation, the simulation results, i.e., cell dose and cell DNA DSB, are used to quantify the cell state transitions. The cell state transition results are used to determine whether cell is killed by radiation or not. If the cell is killed, cell type changes to necrotic. Then the tumor system keeps its evolution path till next irradiation. In this simulation, the total simulation time is $14000 \mathrm{MCS}$, and after simulation, the tumor growth curve can be obtained.

As shown in Figure 6a, six dose schemes are simulated, and the tumor growth curves of those dose schemes are plotted together for comparison. The tumor growth curves show that the tumor growth rate is reduced with the irradiation. The higher the dose, the more the reduction of growth rate. However, interestingly, we can observe that the tumor growth rate under the total dose of 5 Gy in 5 fractions is even higher than the growth rate of no irradiation. The possible reason for this is that the low dose irradiation kills some cells, which eventually leads to more free space for tumor growth since the dead cells will gradually be eliminated in tumor cluster, and this offsets the space constraint due to contact inhibition for cell proliferation.

We also can observe that the number of proliferating tumor cells drop after each irradiation, but tumors will quickly recover from the cell loss when the dose is relatively low in each fraction, which indicates that tumor cell repopulation can offset the tumor cell loss due to irradiation. Besides analyzing the tumor growth curve, we also can observe the morphological change of tumor after irradiation. Through the CC3D simulation, we can visualize the tumor shape with time. For instance, we predict how the tumor cluster will look like after a series of irradiation. Here, we list the snapshots of tumor cluster under four different dose schemes, as shown in Figure 6. 


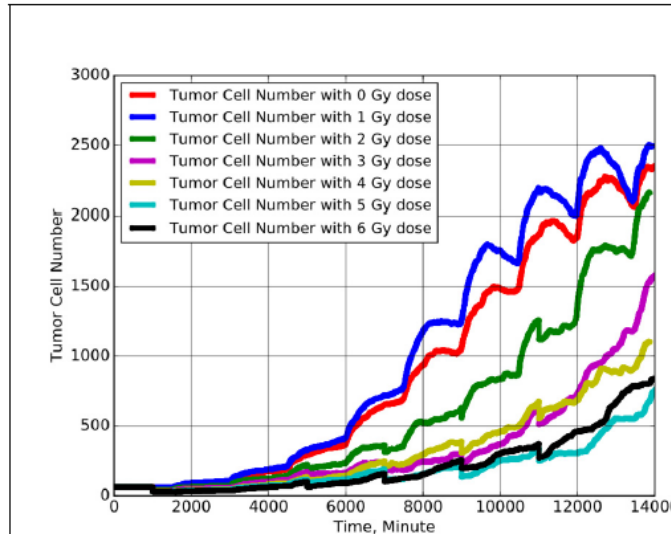

(a) Number of proliferating tumor cells

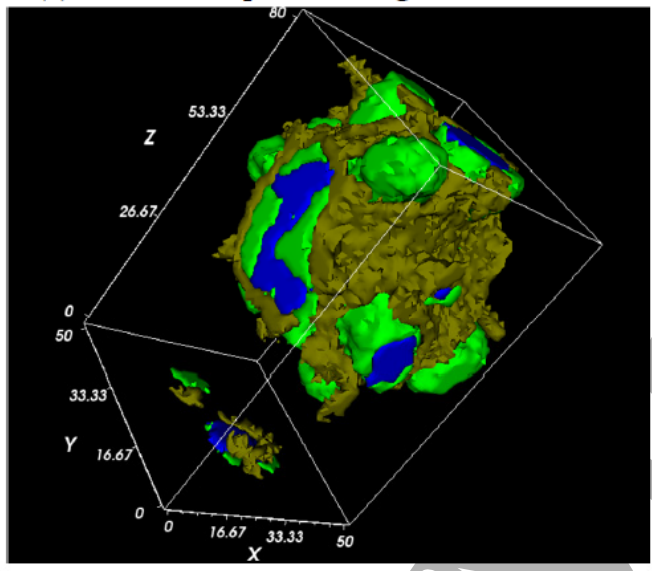

(c) At 14000 MCS after 20 Gy total dose

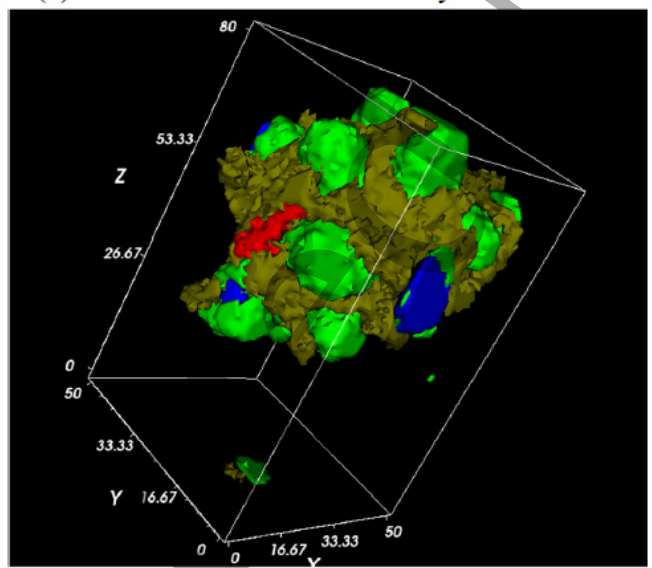

(e) At $14000 \mathrm{MCS}$ after $30 \mathrm{~Gy}$ total dose

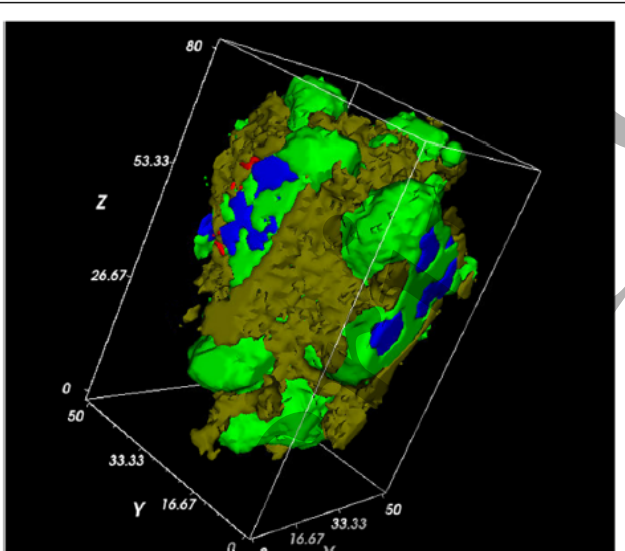

(b) At $14000 \mathrm{MCS}$ after 15 Gy total dose

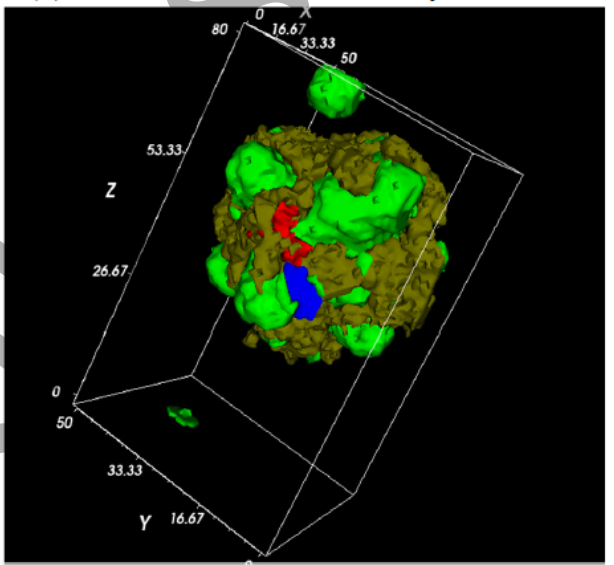

(d) At 14000 MCS after 25 Gy total dose

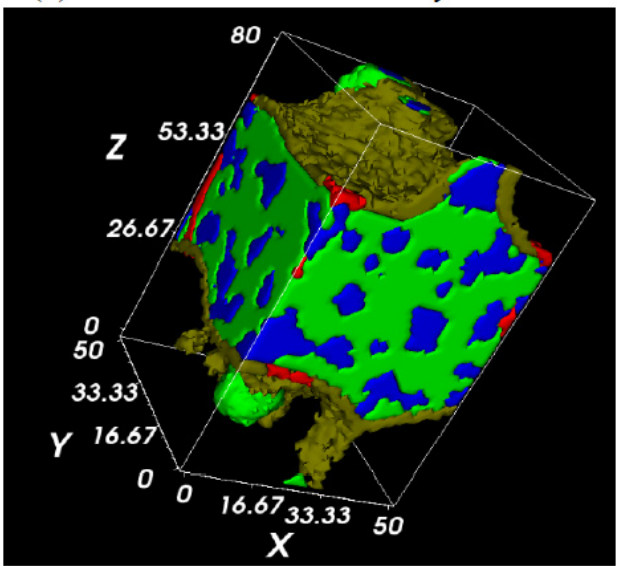

(f) At 14000 MCS without dose

Figure 6: Two-dimensional snapshots of the vascular tumor simulation. Red: vascular cells, grey: neovascular cells, blue: necrotic cells. The spatial unit used in CC3D is pixel, and one pixel equals $4 \mu \mathrm{m}$ in this work. The dose is delivered in 5 fractions, and the delivering time at: 1000 MCS, 5000 MCS, 7000 MCS, 9000 MCS, and 11000 MCS, respectively. Fig. (a) shows the tumor growth curves under different dose levels with respect to tumor evolution time which has MCS as its unit. Fig. (b) shows tumor system after irradiation with 15 Gy dose at 14000 MCS rendering in CC3D visualization system. Figs. (c), (d), (e), and (f) show the tumor 
system after irradiation with $20 \mathrm{~Gy}, 25 \mathrm{~Gy}, 30 \mathrm{~Gy}$, and $0 \mathrm{~Gy}$, respectively. Fig. (f) serves the control compared to the cases with irradiation.

\subsubsection{Tumor Response with Hyperfractionated Dose}

It is found that fractionation of the radiation dose produces, in most cases, better tumor control for a given level of normal tissue toxicity than a single large dose. However, treatment with any cytotoxic agent, including radiation, can trigger surviving cells in a tumor to divide faster than before. The critical point is that during the time that tumor is overtly shrinking and regressing, the surviving clonogens are dividing and increasing in number more rapidly than before treatment [57]. There are two separate strategies to cope with this issue, and they are hyperfractionation and accelerated treatment.

In this simulation, we try to simulate a hyperfractionated dose delivering scheme for vascular tumor. We use a single planar microbeam source to irradiate the vascular tumor cluster. Particularly, the tumor cluster size at the first irradiation is chosen to be relatively large, which is used to model a fully-grown tumor. We obtain this "grown" tumor by letting the initial tumor grow without irradiation for $12000 \mathrm{MCS}$. Two fractionated dose schemes are simulated, the first one is $40 \mathrm{~Gy}$ in five fractions, and the second one is $40 \mathrm{~Gy}$ in 2 fractions.

The dose delivering time for the first scheme is at: 12000 MCS, 13000 MCS, 14000 MCS, 15000 MCS, and 16000 MCS. The dose delivering time for the second scheme is at $12000 \mathrm{MCS}$ and 16000 MCS. For the first scheme, five doses are delivered within 4000 MCS. As we discussed above, 1 MCS approximately equals 1 minute in this study, so we know that roughly there are two doses per day. The first delivering scheme is considered to model a hyperfractionated scheme, and the second scheme is considered to model a hypofractionated scheme.

From the tumor growth curve shown in Figure 7a, we can know that for the first fractionated dose, the hypofractionated scheme leads to a $30.37 \%$ higher tumor cell loss compared to the hyperfractionated scheme, which indicates the higher effectiveness of tumor control by the higher dose. But the hyperfractionated scheme performs better in offsetting the tumor cell repopulation after each fractionated dose. The hypofractionated scheme could not offset the tumor cell repopulation. As shown in Figure 7a, after the first fractionated dose in the hypofractionated scheme, the number of tumor cells gradually recover to almost the initial state by the time of second fractionated dose. This result indicates that the hyperfractionated scheme is better for controlling the vascular tumor recovery under the single planar microbeam irradiation. 
It is worth noting that the tumor cell loss after two hypofractionated dose are different. The second fractionated dose leads to a higher tumor cell loss. The difference could be due to the spatially, biased irradiation and the dynamic tumor cell spatial distribution after irradiation. The irradiation here is a single array microbeam whose beam width is smaller than the dimension size of the simulated tumor, so only a portion of the whole simulated tissue including tumor will be directly irradiated. This can lead to a biased irradiation for the tumor cells that just stay at the irradiated region from the subsequent dose deliveries, especially for the new tumor cells that grow toward to the irradiated region after the first dose delivery. The spatial distribution of tumor cells at these two these dose deliveries is different because the tumor cell spatial distribution is a dynamic process with time. After the first dose delivery, some tumor cells were killed, due to the cell loss, there is more "empty" space for the tumor grow toward to this region. This could lead to more tumor cell killing by the following dose delivery.

The morphological change of tumor after irradiation is shown in Figure $7 \mathrm{~b}$ and Figure $7 \mathrm{c}$. By comparing the shape of the tumor under those two different dose schemes, we can know that both dose schemes can reduce the tumor volume, and the hyperfractionated scheme reduces more tumor volume. 


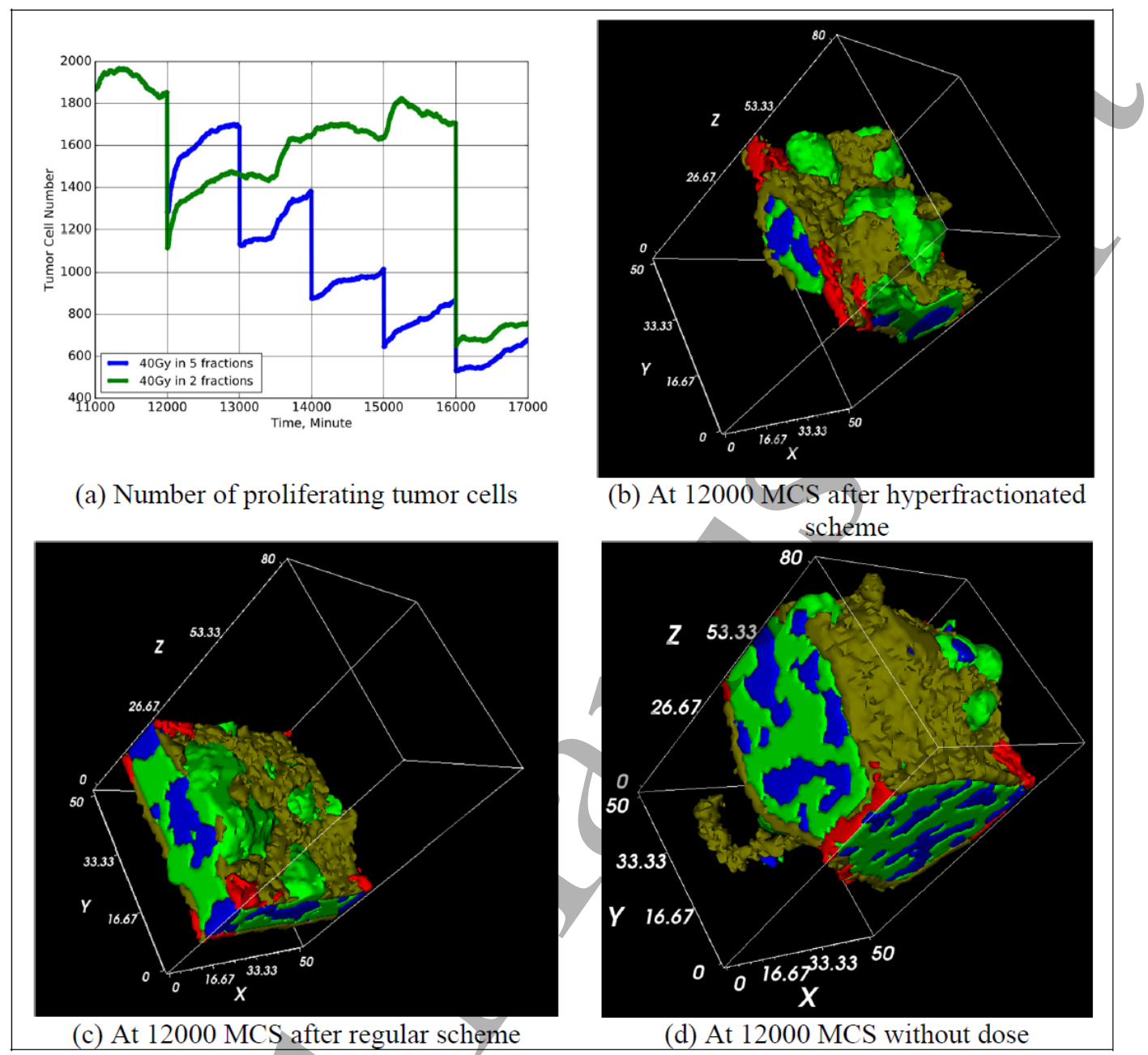

Figure 7: Two-dimensional snapshots of the vascular tumor simulation. Red: vascular cells, grey: neovascular cells, blue: necrotic cells. The spatial unit used in CC3D is pixel, and one pixel equals $4 \mu \mathrm{m}$ in this work. The hyperfractionated doses are delivered at 12000 MCS, $13000 \mathrm{MCS}, 14000 \mathrm{MCS}, 15000 \mathrm{MCS}$, and 16000 MCS. The regular doses are delivered at $12000 \mathrm{MCS}$ and $16000 \mathrm{MCS}$. Fig. (a) shows the tumor growth curve under two different dose deliver schemes. Fig. (b) shows the tumor system at 12000 MCS after the hyperfractionated dose deliver scheme. Fig. (c) shows the tumor system at $12000 \mathrm{MCS}$ after the regular dose deliver scheme. Fig. (d) shows the tumor system at 12000 MCS without irradiation, which serves as control for the cases with irradiation.

\subsubsection{Tumor Response with Fractionated Dose by MRT}

Here, we simulate how the vascular tumor response to the multi-array planar microbeam irradiation. It is worth noting that we use a multi-array microbeam source to achieve the MRT in the real clinical application, so this simulation captures the features of a MRT treatment condition. 
In this simulation, there are five planar microbeam sources separated by $200 \mu \mathrm{m}$ center by center serving as the multi-array microbeam source, as shown in Figure 8. The hyperfractionated dose schemes, i.e., 40 Gy in 5 fractions and 50 Gy in 5 fractions, are selected to deliver the dose to a "grown" vascular tumor. In both schemes, the first irradiation starts at $12000 \mathrm{MCS}$, and dose delivering time is at: $12000 \mathrm{MCS}, 13000 \mathrm{MCS}, 14000 \mathrm{MCS}, 15000 \mathrm{MCS}$, and $16000 \mathrm{MCS}$.

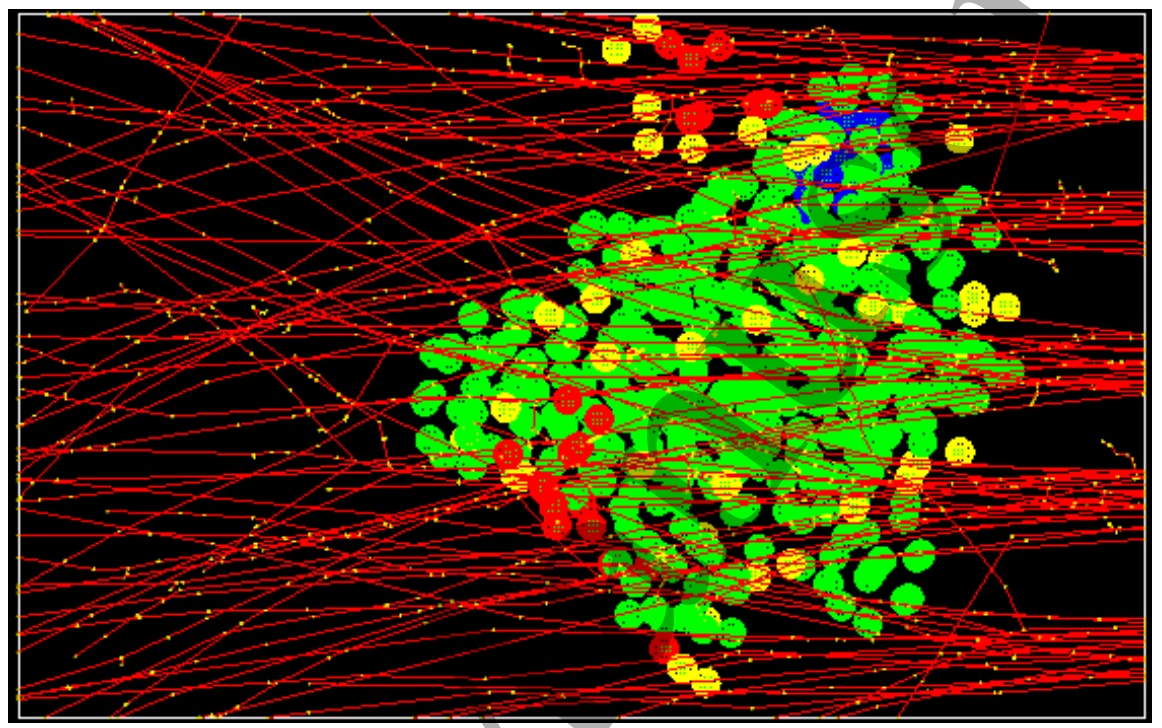

Figure 8: Multi-array planar MRT irradiation on vascular tumor simulated in Geant4. Five panels of planar microbeams are used to irradiate tumor. In Geant4 platform, the red lines are the tracks generated by electrons, and the yellow points are the energy deposition points after radiation interactions.

From the tumor growth curves shown in Figure 9a, we can see that the hyperfractionated scheme by the multi-array microbeam can substantially reduce the growth of tumor cells. After the whole scheme delivery, the proliferating tumor cells are almost eliminated. We can also observe that the two hyperfractionated scheme performs nearly same in terms of the tumor control since, after the whole scheme, all the tumor cells are almost killed. We can calculate the proliferating tumor cell loss after one fraction of the dose by comparing the tumor cell number before and after the dose delivering. As shown in Figure 9a, there is a steep drop in cell number after the delivery of each dose. By comparing the tumor cell loss after each fraction in both fractionated schemes, we can see that there is no substantial difference in those two schemes, which indicates that there is a saturation of tumor control in terms of the total dose. The morphological change of tumor after multi-array planar microbeam irradiation is shown in Figure 9. We can see that after the multiarray microbeam irradiation, the vascular tumor gradually shrinks its volume. 


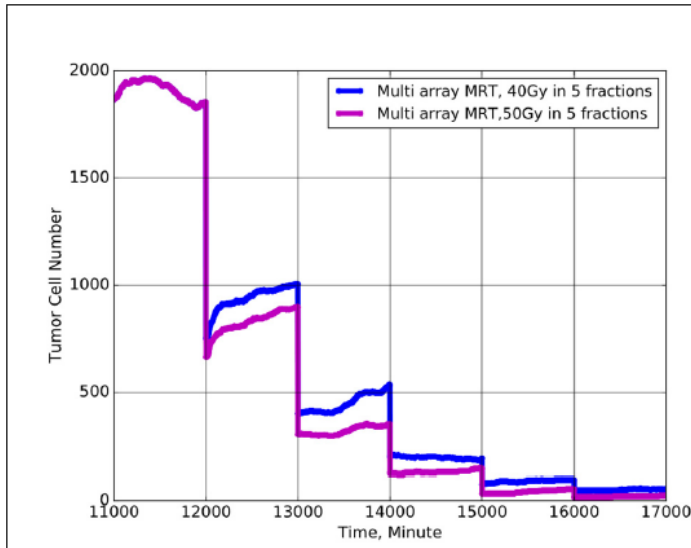

(a) Number of proliferating tumor cells

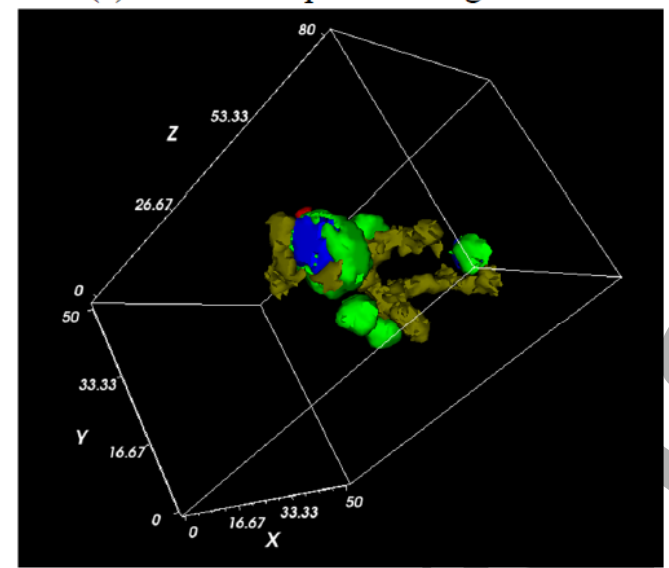

(c) At 17000 MCS after 40 Gy dose

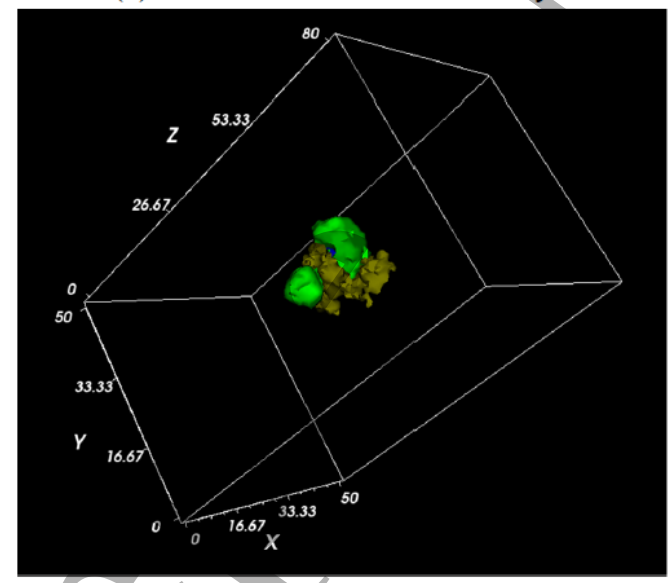

(e) At $17000 \mathrm{MCS}$ after 50 Gy dose

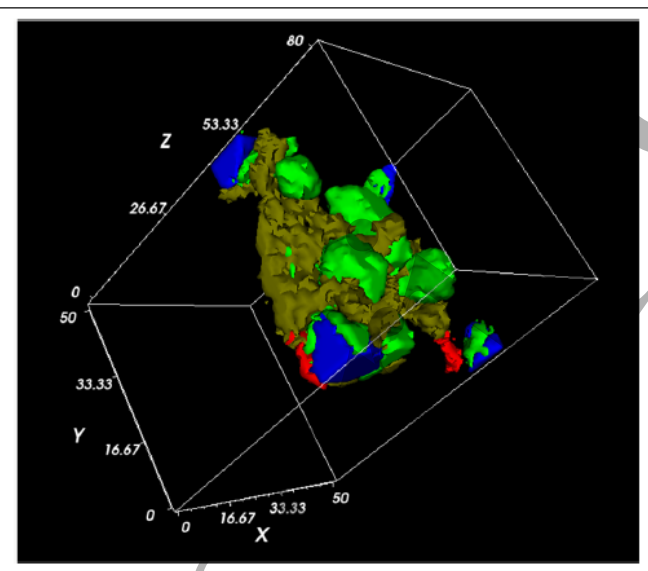

(b) At 15000 MCS after 40 Gy dose

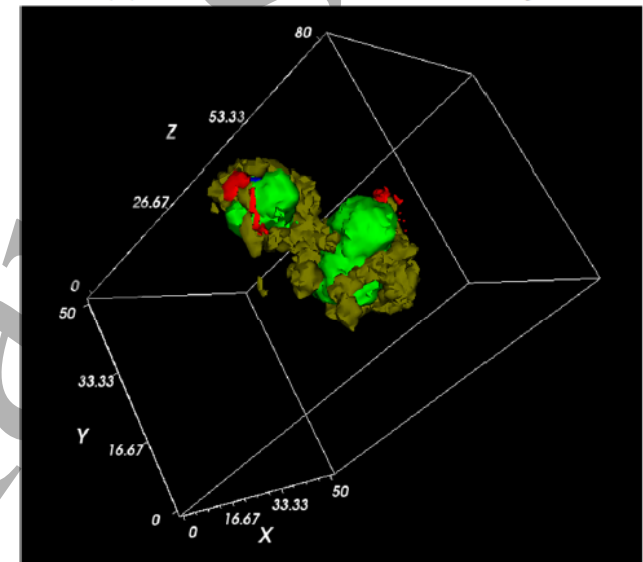

(d) At 15000 MCS after 50 Gy dose

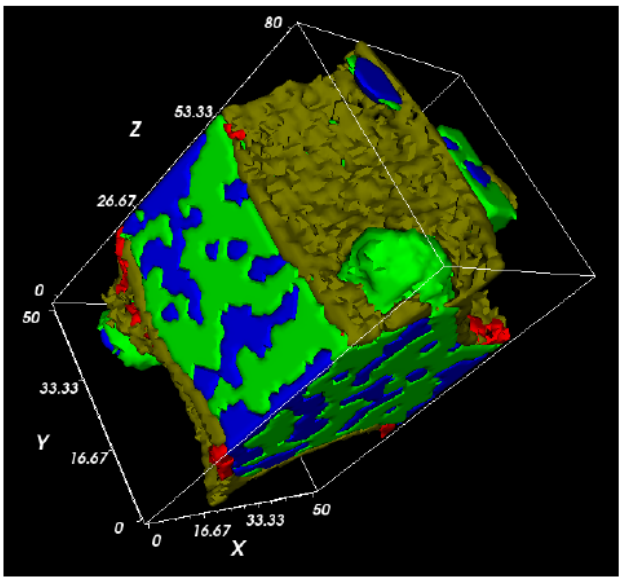

(f) At 17000 MCS without irradiation

Figure 9: Two-dimensional snapshots of the vascular tumor simulation. Red: vascular cells, grey: neovascular cells, blue: necrotic cells. The spatial unit used in CC3D is pixel, and one pixel equals $4 \mu \mathrm{m}$ in this work. The doses are delivered in 5 fractions, and the delivering time at: 12000 MCS, 13000 MCS, 14000 MCS, 15000 MCS, and 16000 MCS, respectively. Fig. (a) shows the tumor growth curve under two different dose deliver schemes. Fig. (b) shows the tumor system at 15000 MCS after irradiation with 40 Gy dose. Fig. (c) shows the 
tumor system at $17000 \mathrm{MCS}$ after irradiation with $40 \mathrm{~Gy}$ dose. Fig. (d) shows the tumor system at $15000 \mathrm{MCS}$ after irradiation with 50 Gy dose. Fig. (e) shows the tumor system at 17000 MCS after irradiation with 50 Gy dose. Fig. (f) shows the tumor system at 17000 MCS without irradiation, which serves the control compared to the cases with irradiation.

\subsection{Future Work}

In this work, we simulate the change of cell-level parameters such as target volume after irradiation by integrating Geant 4 and CC3D. We linked the macroscopic cell behavior according to the cell state transition determined by radiation-induced DSBs and the glucose concentration, but we did not consider linking the macroscopic cell behaviors to the intracellular response. The CC3D framework allows us to add and solve subcellular reaction-kinetic pathway models inside each cell to simulate the cell-level behavior by using SBML [58]. In future study, we can model the cellular response for molecular concentrations that can steer the behaviors of biological cells by modulating their biochemical machinery. For example, we can incorporate the repair pathways, such as NonHomologous End Joining (NHEJ) and Homologous Recombination (HR), by applying the wellknown models [59][60] about DNA damage repair using the intracellular simulation functionality of CC3D. Oxygen is an important factor moderating cellular radiation response. In this work, we did not explicitly model the oxygen diffusion and reaction process between cells and inside cells. Instead, we expect that the tumor cells are usually hypoxic, and they can secrete a long-diffusion isoform of VEGF-A to induce angiogenesis in hypoxic tissue. By using the diffusion solver of CC3D we can simulate the oxygen diffusion and reaction between cells and inside cells. Then we can include the models [61][62] of oxygen effect for simulating the radiation effect on vascular tumor.

\section{Conclusions}

To the best of our knowledge, this multi-platform simulation is the first piece of research to combine Geant4 and CC3D to implement the coupled cell biology and radiation transport simulation for quantifying cell response after irradiation. In this paper, we focused on introducing this novel framework with relevant technical details and illustrated with a case study. A vascular tumor simulation model based on the developed toolkit was studied in this work. The simulation parameters of the vascular tumor model are adopted from previously published research papers. Despite the rescaling of the tumor size, the model produces a range of biologically reasonable morphologies that allow study of how MRT treatment affects the growth rate, size, and morphology of vascular tumors. 
By interacting with clinicians and experimentalists, we may gather experimental data to parameterize, calibrate, and validate the model for its clinical applications.

This method is appealing because it allows quantitative assessment of radiation damage in each cell, allowing us to further build more an informed model of radiation damage in tissue level. The presented method could be validated by performing a quantitative measurement of tumor spheroid subject to precise radiation dose delivery. In addition to controlling radiation dose, we could also vary the temporal pattern of dose delivery. With experimental validation, our developed simulation platform can help to design the effective MRT treatment plan in the real clinical trials in the future. In a broader sense, other type of radiation therapy treatment technique also could be explored in silico through this developed simulation platform. This work shows that the developed model could be potentially used to facilitate the investigation of radiation biology study. We hope that this work shed light on building a comprehensive mathematical modeling toolkit for computational radiation biology.

\section{Acknowledgements}

RL acknowledges support from Consortium for Risk Evaluation and Stakeholder Participation ( http://www.cresp.org). JAG acknowledges support from National Science Foundation grant NSF 1720625 and National Institutes of Health, National Institute of General Medical Sciences grants U01 GM111243 and R01 GM076692, JAG and MS acknowledge support from National Institutes of Health, National Institute of General Medical Sciences grant R01 GM122424. We would like to thank the anonymous reviewers for constructive suggestions for improving this paper.

\section{References}

[1] J. Thariat, J.-M. Hannoun-Levi, A. Sun Myint, T. Vuong, and J.-P. Gérard, "Past, present, and future of radiotherapy for the benefit of patients," Nat. Rev. Clin. Oncol., vol. 10, no. 1, pp. 52-60, 2012, doi: 
10.1038/nrclinonc.2012.203.

[2] W. Friedland and P. Kundrát, "Modeling of Radiation Effects in Cells and Tissues," in Comprehensive Biomedical Physics, vol. 9, 2014, pp. 105-142.

[3] G. Powathil, M. Kohandel, M. Milosevic, and S. Sivaloganathan, "Modeling the spatial distribution of chronic tumor hypoxia: Implications for experimental and clinical studies," Comput. Math.Methods Med., vol. 2012, pp. 1-11, 2012, doi: 10.1155/2012/410602.

[4] M. J. Ibahim et al., "An evaluation of dose equivalence between synchrotron microbeam radiation therapy and conventional broadbeam radiation using clonogenic and cell impedance assays," PLoS One, vol. 9, no. 6, p. e100547, Jun. 2014, doi: 10.1371/journal.pone.0100547.

[5] M. Tomezak, C. Abbadie, E. Lartigau, and F. Cleri, "A biophysical model of cell evolution after cytotoxic treatments: damage, repair and cell response,"J. Theor. Biol., vol. 389, pp. 146-158, 2015, doi: 10.1016/j.jtbi.2015.10.017.

[6] Y. Hattori, A. Yokoya, and R. Watanabe, "Cellular automaton-based model for radiation-induced bystander effects.," BMC Syst. Biol., vol. 9, no. 1, p. 90, Jan. 2015, doi: 10.1186/s12918-015-0235-2.

[7] V. A. Semenenko and R. D. Stewart, "Fast Monte Carlo simulation of DNA damage formed by electrons and light ions," Phys. Med. Biol., vol. 51, pp. 1693-1706, 2006, doi: 10.1088/00319155/51/7/004.

[8] R. Liu, T. Zhao, X. Zhao, and F. J. Reynoso, "Modeling Gold Nanoparticle Radiosensitization using a Clustering Algorithm to Quantify DNA Double-Strand Breaks with Mixed-Physics Monte Carlo Simulation," Med. Phys., Sep. 2019, doi: 10.1002/mp.13813.

[9] W. Wang et al., "Modelling of Cellular Survival Following Radiation-Induced DNA Double-Strand Breaks," Sci. Rep., vol. 8, no. 1, pp. 1-12, Dec. 2018, doi: 10.1038/s41598-018-34159-3.

[10] W. Friedland, M. Dingfelder, P. Kundrát, and P. Jacob, "Track structures, DNA targets and radiation effects in the biophysical Monte Carlo simulation code PARTRAC," Mutation Research -

Fundamental and Molecular Mechanisms of Mutagenesis, vol. 711, no. 1-2. Elsevier, pp. 28-40, Jun. 03, 2011, doi: 10.1016/j.mrfmmm.2011.01.003.

[11] R. D. Stewart et al., "Rapid MCNP simulation of DNA double strand break (DSB) relative biological effectiveness (RBE) for photons, neutrons, and light ions," Phys. Med. Biol., vol. 60, no. 21, pp. 8249-8274, Oct. 2015, doi: 10.1088/0031-9155/60/21/8249.

[12] G. Baiocco et al., "The origin of neutron biological effectiveness as a function of energy," Sci. Rep., vol. 6, no. 1, pp. 1-14, Sep. 2016, doi: 10.1038/srep34033. 
[13] A. Manuscript, Multi-Scale Modeling of Tissues Using CompuCell3D, vol. 29, no. 1. 2012.

[14] S. Agostinelli et al., "GEANT4 - A simulation toolkit," Nucl. Instruments Methods Phys. Res. Sect. A Accel. Spectrometers, Detect. Assoc. Equip., vol. 506, no. 3, pp. 250-303, Jul. 2003, doi: 10.1016/S0168-9002(03)01368-8.

[15] J. Poleszczuk and H. Enderling, "A High-Performance Cellular Automaton Model of Tumor Growth with Dynamically Growing Domains.," Appl. Math., vol. 5, no. 1, pp. 144-152, 2014, doi: 10.4236/am.2014.51017.

[16] A. R. A. Anderson, M. A. J. Chaplain, and K. A. Rejniak, Single-cell-based models in biology and medicine. Birkhäuser, 2007.

[17] D. Drasdo, "Center-based Single-cell Models: An Approach to Multi-cellular Organization Based on a Conceptual Analogy to Colloidal Particles," in Single-Cell-Based Models in Biology and Medicine, Basel: Birkhäuser Basel, pp. 171-196.

[18] Glazier and Graner, "Simulation of the differential adhesion driven rearrangement of biological cells.," Phys. Rev. E. Stat. Phys. Plasmas. Fluids. Relat. Interdiscip. Topics, vol. 47, no. 3, pp. 2128 2154, Mar. 1993, Accessed: Dec. 04, 2017. [Online]. Available: http://www.ncbi.nlm.nih.gov/pubmed/9960234.

[19] T. J. Newman, "Modeling multicellular systems using subcellular elements.," Math. Biosci. Eng., vol. 2, no. 3, pp. 613-24, Jul. 2005, Accessed: Dec. 04, 2017. [Online]. Available: http://www.ncbi.nlm.nih.gov/pubmed/20369943.

[20] E. T. Somogyi et al., "LibRoadRunner: A high performance SBML simulation and analysis library," Bioinformatics, vol.31, no. 20, pp. 3315-3321, Oct. 2015, doi: 10.1093/bioinformatics/btv363.

[21] J. M. Belmonte et al., "Virtual-tissue computer simulations define the roles of cell adhesion and proliferation in the onset of kidney cystic disease," Mol. Biol. Cell, vol. 27, no. 22, pp. 3673-3685, Nov. 2016, doi: 10.1091/mbc.E16-01-0059.

[22] M. H. Swat, G. L. Thomas, A. Shirinifard, S. G. Clendenon, and J. A. Glazier, "Emergent stratification in solid tumors selects for reduced cohesion of tumor cells: A multi-cell, virtual-tissue model of tumor evolution using CompuCel13D," PLoS One, vol. 10, no. 6, p. e0127972, 2015, doi: 10.1371/journal.pone.0127972.

[23] A. Shirinifard et al., "Adhesion failures determine the pattern of choroidal neovascularization in the eye: A computer simulation study," PLoS Comput. Biol., vol. 8, no. 5, 2012, doi: 10.1371/journal.pcbi.1002440. 
[24] A. S. Maciej H. Swat, Julio Belmonte, Randy W. Heiland, Benjamin L. Zaitlen, James A. Glazier, "CompuCell3D Reference Manual Version 3.7.4," 2016.

[25] M. H. Swat, J. M. Belmonte, R. W. Heiland, B. L. Zaitlen, J. A. Glazier, and A. Shirinifard, "Introduction to CompuCell3D Version 3.7.4," 2016.

[26] M. H. Swat, J. M. Belmonte, and B. L. Zaitlen, "CompuCell3D Python Scripting Manual Version 3.7.4," 2016.

[27] M. H. Swat, G. L. Thomas, J. M. Belmonte, A. Shirinifard, D. Hmeljak, and J. A. Glazier, "Multiscale modeling of tissues using CompuCell3D.," Methods Cell Biot., vol. 110, pp. 325-66, Jan. 2012, doi: 10.1016/B978-0-12-388403-9.00013-8.

[28] M. Šefl, S. Incerti, G. Papamichael, and D. Emfietzoglou, "Calculation of cellular S-values using Geant4-DNA: The effect of cell geometry," Appl. Radiat. Isot., vol. 104, pp. 113-123, 2015, doi: 10.1016/j.apradiso.2015.06.027.

[29] J. Sander, M. Ester, H.-P. Kriegel, and X. Xu, "A Density-Based Algorithm for Discovering Clusters in Large Spatial Databases with Noise," Data Min. Knowl. Discov., vol. 2, no. 2, pp. 169-194, 1998, doi: 10.1023/A:1009745219419.

[30] M. Dos Santos, C. Villagrasa, I. Clairand, and S. Incerti, "Influence of the DNA density on the number of clustered damages created by protons of different energies," Nucl. Instruments Methods Phys. Res. Sect. B Beam Interact. with Mater. Atoms, vol. 298, pp. 47-54, Mar. 2013, doi: 10.1016/j.nimb.2013.01.009.

[31] M. Douglass, E. Bezak, and S. Penfold, "Development of a radiation track structure clustering algorithm for the prediction of DNA DSB yields and radiation induced cell death in Eukaryotic cells," Phys. Med. Biol., vol. 60, no. 8, pp. 3217-3236, 2015, doi: 10.1088/0031-9155/60/8/3217.

[32] Z. Francis, C. Villagrasa, and I. Clairand, "Simulation of DNA damage clustering after proton irradiation using an adapted DBSCAN algorithm," Comput. Methods Programs Biomed., vol. 101, no. 3, pp. 265-270, 2011, doi: 10.1016/j.cmpb.2010.12.012.

[33] R. Liu, T. Zhao, M. H. Swat, F. J. Reynoso, and K. A. Higley, "Development of Computational Model for Cell Dose and DNA Damage Quantification of Multicellular System,” Int. J. Radiat. Biol., pp. 114, Jul. 2019, doi: 10.1080/09553002.2019.1642537.

[34] R. Liu, "Integrated Spatial and Temporal Stochastic Model in Radiation Biology:Design, Implementation, and Application,” Oregon State University, 2017.

[35] W. Friedland, M. Dingfelder, P. Kundrát, and P. Jacob, "Track structures, DNA targets and radiation 
effects in the biophysical Monte Carlo simulation code PARTRAC.," Mutat. Res., vol. 711, no. 1-2, pp. 28-40, 2011, [Online]. Available: http://www.ncbi.nlm.nih.gov/pubmed/21281649.

[36] W. Friedland, E. Schmitt, P. Kundrát, G. Baiocco, and A. Ottolenghi, "Track-structure simulations of energy deposition patterns to mitochondria and damage to their DNA," Int. J. Radiat. Biol., vol. 95, no. 1, pp. 3-11, 2018, doi: 10.1080/09553002.2018.1450532.

[37] W. W. Y. Kam and R. B. Banati, "Effects of ionizing radiation on mitochondria," Free Radic. Biol. Med., vol. 65, pp. 607-619, 2013, doi: 10.1016/j.freeradbiomed.2013.07.024.

[38] N. Albright, "A Markov Formulation of the Repair-Misrepair Model of Cell Survival," Radiat. Res., vol. 118, no. 1, pp. 1-20, 1989.

[39] M. P. Little, J. A. Filipe, K. M. Prise, M. Folkard, and O. V Belyakov, "A model for radiation-induced bystander effects, with allowance for spatial position and the effects of cell turnover," $J$ Theor Biol, vol. 232, no. 3, pp. 329-338, 2005, doi: 10.1016/j.jtbi.2004.08.016.

[40] F. P. Faria, R. Dickman, and C. H. C. Moreira, "Models of the radiation-induced bystander effect.," Int. J. Radiat. Biol., vol. 88, no. 8, pp. 592-9, Aug. 2012, doi: 10.3109/09553002.2012.692568.

[41] J. Von, N. Sujnjnary, and G. E. Forsythe, "Various Techniques Used in Connection With Random Digits," Natl. Bur. Stand. Appl. Math Ser., no. 12, pp. 36-38, 1951, Accessed: Jun. 04, 2018. [Online]. Available: https://mcnp.lanl.gov/pdf_files/nbs_vonneumann.pdf.

[42] K. Murakami and H. Yoshida, "A geant4-python interface: Development and its applications," in IEEE Nuclear Science Symposium Conference Record, 2006, vol. 1, pp. 98-100, doi: 10.1109/NSSMIC.2006.356115.

[43] D. M. Beazley, "Automated scientific software scripting with SWIG," Futur. Gener. Comput. Syst., vol. 19, no. 5 SPEC, pp. 599-609, Jul. 2003, doi: 10.1016/S0167-739X(02)00171-1.

[44] A. Shirinifard et al., "3D Multi-Cell Simulation of Tumor Growth and Angiogenesis," PLoS One, vol. 4, no. 10,2009, doi: 10.1371/journal.pone.0007190.

[45] Y. T. Nguyen Edalgo, A. L. Zornes, and A. N. Ford Versypt, "A hybrid discrete-continuous model of metastatic cancer cell migration through a remodeling extracellular matrix," AIChE J., vol. 65, no. 9, Sep. 2019, doi: 10.1002/aic. 16671.

[46] A. Al-Ramadan, A. C. Mortensen, J. Carlsson, and M. V. Nestor, "Analysis of radiation effects in two irradiated tumor spheroid models," Oncol. Lett., vol. 15, no. 3, pp. 3008-3016, 2018, doi: 10.3892/ol.2017.7716. 
[47] S. C. Brüningk, P. Ziegenhein, I. Rivens, U. Oelfke, and G. ter Haar, "A cellular automaton model for spheroid response to radiation and hyperthermia treatments," Sci. Rep., vol. 9, no. 1, pp. 1-12, 2019, doi: 10.1038/s41598-019-54117-x.

[48] S. C. Brüningk, I. Rivens, C. Box, U. Oelfke, and G. ter Haar, "3D tumour spheroids for the prediction of the effects of radiation and hyperthermia treatments," Sci. Rep., vol. 10, no. 1, pp. 1-13, 2020, doi: 10.1038/s41598-020-58569-4.

[49] M. A. Grotzer, E. Schültke, E. Bräuer-Krisch, and J. A. Laissue, "Microbeam radiation therapy: Clinical perspectives," Phys. Medica, vol. 31, no. 6, pp. 564-567, 2015, doi: 10.1016/j.ejmp.2015.02.011.

[50] E. C. Schreiber and S. X. Chang, "Monte Carlo simulation of a compact microbeam radiotherapy system based on carbon nanotube field emission technology," Med. Phys., vol. 39, no. 8, pp. 46694678, 2012, doi: 10.1118/1.4728220.

[51] E. Bräuer-Krisch et al., "Effects of pulsed, spatially fractionated, microscopic synchrotron X-ray beams on normal and tumoral brain tissue," Mutation Research - Reviews in Mutation Research, vol. 704, no. 1-3. Elsevier, pp. 160-166, Apr. 01, 2010, doi: 10.1016/j.mrrev.2009.12.003.

[52] E. Bräuer-Krisch et al., "Medical physics aspects of the synchrotron radiation therapies: Microbeam radiation therapy (MRT) and synchrotron stereotactic radiotherapy (SSRT)," Phys. Medica, vol. 31, no. 6, pp. 568-583, 2015, doi: 10.1016/j.ejmp.2015.04.016.

[53] J. Spiga, E. A. Siegbahn, E. Bräuer-Krisch, P. Randaccio, and A. Bravin, "Geant4 simulations for Microbeam Radiation Therapy (MRT) dosimetry," IEEE Nucl. Sci. Symp. Conf. Rec., vol. 4, pp. 2571-2575, 2007, doi: 10.1109/NSSMIC.2007.4436675.

[54] H. Blattmann et al., "Applications of synchrotron X-rays to radiotherapy," in Nuclear Instruments and Methods in Physics Research, Section A: Accelerators, Spectrometers, Detectors and Associated Equipment, Aug. 2005, vol. 548, no. 1-2, pp. 17-22, doi: 10.1016/j.nima.2005.03.060.

[55] a L. McNamara, U. Oelfke, and Z. Kuncic, "Revealing the underlying mechanism of microbeam radiation therapy with low energy Monte Carlo simulations," J. Phys. Conf. Ser., vol. 489, no. 1, p. 012018, 2014, doi: 10.1088/1742-6596/489/1/012018.

[56] E. A. Siegbahn, E. Bräuer-Krisch, J. Stepanek, H. Blattmann, J. A. Laissue, and A. Bravin, "Dosimetric studies of microbeam radiation therapy (MRT) with Monte Carlo simulations," Nucl. Instruments Methods Phys. Res. Sect. A Accel. Spectrometers, Detect. Assoc. Equip., vol. 548, no. 1-2, pp. 54-58, 2005, doi: 10.1016/j.nima.2005.03.065. 
[57] E. J. Hall and A. J. Giaccia, Radiobiology for The Radiologist, 7th ed. 2012.

[58] M. Hucka et al., "The systems biology markup language (SBML): A medium for representation and exchange of biochemical network models," Bioinformatics, vol. 19, no. 4, pp. 524-531, 2003, doi: 10.1093/bioinformatics/btg015.

[59] S. P. Ingram et al., "Mechanistic modelling supports entwined rather than exclusively competitive DNA double-strand break repair pathway," Sci. Rep., vol. 9, no. 1, p. 6359, Dec. 2019, doi: 10.1038/s41598-019-42901-8.

[60] J. W. Warmenhoven et al., “An In Silico Model of DNA Repair for Investigation of Mechanisms in Non-Homologous End Joining,” bioRxiv, p. 318139, 2018, doi: 10.1101/318139.

[61] D. J. Carlson, R. D. Stewart, and V. A. Semenenko, "Effects of oxygen on intrinsic radiation sensitivity: A test of the relationship between aerobic and hypoxic linear-quadratic (LQ) model parameters," in Medical Physics, 2006, vol. 33, no. 9, pp. 3105-3115, doi: 10.1118/1.2229427.

[62] R. D. Stewart, V. K. Yu, A. G. Georgakilas, C. Koumenis, J. H. Park, and D. J. Carlson, "Effects of Radiation Quality and Oxygen on Clustered DNA Lesions and Cell Death," Radiat. Res., vol. 176, no. 5, pp. 587-602, Nov. 2011, doi: 10.1667/rr2663.1. 